BONPLANDIA 20(2). 2011

ISSN: 0524-0476

\title{
PROBLEMAS DE LA ETNOTAXONOMÍA GUARANÍ: "LAS PLANTAS DE LOS ANIMALES"
}

\author{
HÉCTOR A. KELLER ${ }^{1}$
}

\begin{abstract}
Summary: Keller, H.A. 2011. Problems of guarani ethnotaxonomy: "Animals plants". Bonplandia 20(2): 111-136.

Results of a research on certain guarani plant names are presented. These names, very abundant in the ethnographical, ethnobotanical and ethnopharmacological literature, refer to "the plant that belong to the animals" and constitute a dilemma not resolved in preceding contributions. The methodological perspective used corresponds to the ethnographical focusing, with certain variants related of the reflexive trajectory of the author. The current cosmological premises of the guaranis, that determine the kind of the plant names mentioned, are capable of stumped the frontier between "which it is " and " which is not " a plant, returning inapplicable for this investigation the ontological fears of the ethnotaxonomy.
\end{abstract}

Key words: Plant names, Mbya, ontology, mythology, aromatic plants.

Resumen: Keller, H.A. 2011. Problemas de la etnotaxonomía guaraní: "Las plantas de los animales". Bonplandia 20(2): 111-136.

Se presentan los resultados de una investigación sobre ciertos fitónimos guaraníes muy abundantes en la literatura etnográfica, etnobotánica y etnofarmacológica. Estos hacen referencia a las "plantas que pertenecen a los animales", y constituyen un dilema que se presenta como irresuelto en contribuciones precedentes. La perspectiva metodológica empleada se inscribe en el enfoque etnográfico, con ciertas variantes propias de la trayectoria reflexiva del autor. Los fundamentos guaraníes vigentes, de índole cosmológica, que determinan la naturaleza de los fitónimos mencionados, son capaces de difuminar la frontera entre "lo que es" y "lo que no es" una planta, tornando inaplicables para esta investigación las aprensiones ontológicas de la Etnotaxonomía.

Palabras clave: Fitónimos, Mbya, ontología, mitología, plantas aromáticas.

\section{Introducción}

En diversas regiones del globo las nomenclaturas populares han nombrado plantas sobre la base de atributos que denotan su pertenencia a los animales. Dicha relación de pertenencia generalmente se asocia al empleo supuesto o fehaciente de materiales o sustancias vegetales por parte de ciertos representantes de la fauna silvestre o doméstica; se citan algunos ejemplos. Según Montenegro (2007), en Tucumán llamaban a cierta planta "la yerba del hurón" pues se sugería que dicho mamífero la ingiere para tratarse cuando es mordido por una serpiente y se revuelca sobre la planta, para atontar con su olor a las serpientes, antes de matarlas (p. 146). La "yerba de la golondrina" es un fitónimo

${ }^{1}$ Investigador del CONICET, Instituto de Botánica del Nordeste, UNNE, Casilla de correo 209, 3400 Corrientes, Argentina; kellerhector@hotmail.com. 
recurrente y muy antiguo en la medicina oftalmológica popular hispanoamericana (Ruiz, 1952) considerándose que su látex es usado por las golondrinas para "curar los ojos" de sus pichones, quienes a la hora de abandonar el nido deben contar con una visión lo suficientemente aguda como para capturar insectos en vuelo; dicha aplicación ha sido incluida en la conformación de la denominación varietal de la planta; Euphorbia hirta var. ophtalmica (Euphorbiaceae). Pero este fitónimo parece provenir del "viejo mundo" ya que en Inglaterra denominan "swallow wort" (hierba de la golondrina) a una asclepiadácea, también latescente, Vincetoxicum hirundinaria (Podlech, 2001), cuyo epíteto específico hace referencia al género "Hirundo" al cual pertenecen muchas golondrinas. En el sur mendocino llaman "pasto perro" a la especie Bromus catharticus (Poaceae), haciendo probable referencia al uso que hacen de ella los perros para mejorar sus procesos digestivos (Muiño et al., 2006). Varios de los nombres asignados a las "plantas de los animales" conforman parte de la nomenclatura científica, habiéndose formulado epítetos tales como apianus: "de las abejas"; avium: "de las aves"; formicarius: "de las hormigas"; perdicarius: "de las perdices" (SterndaleBennett, 2005).

Las especies que los guaraníes consideran "plantas de los animales" se designan mediante binomios compuestos por el sufijo " $k a$ ' $a$ " (planta, hierba) precedido por el nombre del animal al cual presuntamente pertenece la planta (ej. inambu ka'a: la hierba de la perdiz). Los primeros registros escritos por los jesuitas sobre la lengua guaraní (Montoya, 1639) ya daban cuenta de expresiones tales como "mbói chini ka'a" (la hierba de la serpiente de cascabel) y "makagua ka' $a$ " (la hierba de cierta especie de ave). En el año 1710 otro integrante de la "Compañía de Jesús" mencionaba en su obra sobre materia médica misionera no menos de una decena de fitónimos guaraníes que hacen referencia a "plantas de animales", incluyendo algunas que aluden a especies animales alóctonas como "kavara ka'a" (la hierba de la cabra) y "toro $k a$ 'a" (la hierba del toro) (Montenegro, 2007). Desde entonces y hasta la actualidad este tipo de fitónimos zoológicos guaraníes ha sido de aparición recurrente en documentos escritos por misioneros, cronistas de viajes, estudiosos y científicos (Bertoni, 1940; Balée \& Cebolla Badie, 2009; Cadogan, 1957; Crivos et al., 2007; Hieronymus, 1882; Martínez Crovetto, 1980; Müller, 1935; Queirel, 1897; Tastevin, 1923, entre muchos otros), siendo de uso muy reiterado en tratados sobre la farmacopea argentina y latinoamericana (Alonso \& Desmarchelier, 2005; Amat \& Yajía, 1998; Barboza et al., 2009; Chiarini, 1975; Gupta, 1995; Kozel, 1965; Lahitte \& Hurrel, 1994). Dada su abundancia y popularidad, es posible que estos fitónimos zoológicos guaraníes se hayan infiltrado también en la nomenclatura científica. El epíteto "ipecacuanha" ha sido asignado a una planta del género Psychothria L. (Rubiaceae) denominada "ipeca" en lengua tupi-guaraní del Brasil (Torres Montes, 1998), habiéndose propuesto varias implicaciones etimológicas para dicho fitónimo; pero es muy probable que el término provenga de "ipe $k a$ 'a" (la hierba del pato), ya que esa misma expresión tupí guaraní o su traducción literal, también se aplican a congéneres de la especie vegetal mencionada y a otros representantes de la misma familia de plantas (cfr. Balée, 1994; Marzocca, 1976).

Las etnociencias han desarrollado disciplinas que se instituyen como competentes para abordar la nomenclatura biológica tradicional; la Clasificación Folk da cuenta de la vía por la cual los miembros de una comunidad lingüística nominan y categorizan a plantas y animales (Brown, 2000) en tanto la Taxonomía Folk o Etnotaxonomía es una disciplina que aborda la estructura jerárquica, contenido orgánico y función cultural de las clasificaciones biológicas con las que los etnobiólogos se encuentran en las sociedades que pueblan el planeta (Atran et al., 2004). Las corrientes etnotaxonómicas guardan una estrecha relación con la taxonomía y la sistemática biológica, cuyos objetivos consisten en ordenar la enorme diversidad de organismos en sus distintas formas $y$ maneras de vivir; describir las especies, darles nombres, clasificarlas y reflejar las relaciones naturales de parentesco entre los organismos y los grupos de organismos 
(Strasburger et al., 2003). Estas ramas de la biología, al amalgamarse a los encuadres auspiciosos de corrientes antropológicas estructuralistas y universalistas ${ }^{1}$, inspiraron el desarrollo de disciplinas encargadas de abordar la nomenclatura biológica tradicional. Uno de sus más conocidos exponentes, Berlin, (1973) llamaba la atención sobre ciertas regularidades tipológicas y estructurales en la categorización y nomenclatura de los sistemas de clasificación etnobiológicos a lo largo de todo el mundo. En concordancia con dichas regularidades, el autor sugería que las taxonomías tradicionales presentan grados de equivalencia con la taxonomía moderna.

Pero el estudio de la nomenclatura guaraní ya se había adelantado varias décadas a este postulado universalista. En 1939, Dennler señalaba algunas semejanzas entre la nomenclatura científica y las denominaciones zoológicas guaraníes de la Argentina y países limítrofes. Un año más tarde, Moisés Bertoni (1940), luego de haber compilado y clasificado un gran número de nombres de plantas, adjudicaba a los guaraníes "la sola cientifica de todas las nomenclaturas vulgares existentes", sugiriendo que se trataría de una nomenclatura binomial, donde el género y la especie aparecen de manera inconfundible, encontrándose usualmente también el concepto de familia natural. Dicho autor también afirmaba que los "guaraníes de raza" rinden culto a la nomenclatura y gestan los nombres de plantas y animales en asambleas igualitarias y democráticas. Sin embargo, una de las categorías que más importunaba a este autor en su intento de sugerir una nomenclatura guaraní binomial y jerárquica (familia-géneroespecie) fue justamente la de los nombres de plantas que denotan su pertenencia a animales. Como salvaguarda reflexionaba sobre el valor etnoecológico de dichos fitónimos: "al que no esté muy al tanto, parecerá contradecir a todo esto, el uso frecuente de nombres de animales que aparentemente no pueden venir al caso.... La reflexión puede, sin embargo, explicar el enigma cuando la información directa con los indígenas no es posible.
Pues aquellos nombres indican un carácter ecológico en la gran mayoría de los casos, es decir que designan a la planta por el medio en que vive, y este medio es designado a su vez por el nombre de un animal que en ese medio frecuenta. Así por ejemplo teyú kaá = "la yerba del lagarto" es nombre de plantas que suelen crecer en los lugares asoleados y pedregosos, donde también abundan los lagartos". (P. 152-153)

Pero, aunque no se ajusten a las expectativas clasificatorias binomiales y jerárquicas propuestas por dicho autor, las expresiones que aluden a "las plantas de los animales" se aplican a una importante fracción de los fitónimos guaraníes. Un cuadro comparativo efectuado por Martínez Crovetto (1968) permite apreciar que entre los indígenas del nordeste argentino, son los guaraníes (Mbya) quienes aplican el mayor número de fitónimos referentes al "uso real o supuesto que hacen los animales" de las especies vegetales, alcanzando éstos el 17\% de los nombres registrados por el autor. Según Cadogan (1957), los "nombres zoológicos" de las plantas superan el $25 \%$ de la nomenclatura mbya guaraní, ya que todo miembro del reino animal poseería una hierba que le es de su pertenencia. El autor complementaba dicha aseveración con un relato del folklore paraguayo en el cual se dice que el Creador habría asignado a cada animal una hierba con la cual curarse.

Más allá de este relato con matices de sincretismo cristiano-guaraní y de las consideraciones etnoecológicas de Bertoni (1940), las razones por las cuales para los guaraníes ciertas plantas pertenecen a los animales permanecieron hasta el presente sin esclarecerse. Sin embargo la etnobotánica proporcionó unas pocas pistas conducentes. Las únicas tres especies vegetales registradas para la magia amorosa de un grupo tupí guaraní amazónico son aromáticas y sus nombres expresan la pertenencia a ciertos peces (Balée, 1994). Cadogan (1968) notó que algunas de las, llamadas por los Mbya del Paraguay, "plantas de los animales" son aromáticas y se usan como amuletos o filtros.

${ }^{1}$ Las taxonomías de las diferentes culturas forman parte de una taxonomía global y dinámica, cuya estructura es perfectamente homogénea (Levi Straus, 1964) 
Asimismo, Keller (2007a) tras un estudio etnobotánico llevado a cabo en varias aldeas guaraníes de Misiones, Argentina, señaló que las plantas mágicas usadas por sus integrantes poseen generalmente nombres asociados a animales y muchas veces son aromáticas. Entre los guaraníes Mbya y Ava Chiripa de un sector de Santa Catarina, Brasil se han registrado algunas "plantas de los animales" que se emplean para fines mágico-religiosos (Oliveira, 2009). Por su parte, contribuciones sobre otros grupos indígenas de Argentina también han señalado fuertes vinculaciones de la magia amorosa con las plantas aromáticas y con especies vegetales que constituyen los elementos simbólicos de determinados animales (Arenas \& Braunstein, 1981).

El conjunto de las vicisitudes expuestas conformaron el sustrato elemental sobre el cual ciertos interrogantes sencillos pero latentes, germinaron y se establecieron como preguntas recurrentes en todas las jornadas de diálogo con interlocutores guaraníes llevadas a cabo desde inicios del corriente año, ¿Cómo es eso de que ciertas plantas son propiedad de los animales? ¿Cómo es que llegaron a poseerlas? ¿Porqué son tan usadas en procedimientos mágicos para atraer al sexo opuesto?. La presente contribución pivota sobre las respuestas obtenidas.

\section{Contexto biocultural}

Los guaraníes meridionales constituyen un grupo étnico de origen amazónico que se estableció en su actual área de distribución hace algo más de dos milenios (Schmitz, 1991). Su población contemporánea se compone de al menos 65.000 individuos, constituyéndose en una de las mayores poblaciones indígenas de las tierras bajas de América del Sur (Assis \& Garlet, 2004). Han desarrollado una notable agricultura que data de la época de su arribo a esta región (Schmitz, 1991), contando hasta la actualidad con una amplia gama de cultígenos (Cámara Hernández \& Miante Alzogaray, 2011; Utermoehl \& Gonçalves, 2007). Complementariamente han sabido obtener parte de sus recursos proteínicos a partir de la caza, la pesca, la cría de larvas y las prácticas pecuarias incorporadas después de la "colonización". Su intensa relación con las plantas ha sido explicada como consecuencia de la biodiversidad vegetal del medio natural en el que viven (Martínez Crovetto, 1968).

La población guaraní en la provincia de Misiones supera los 5500 individuos, la mayoría de ellos pertenecientes a la parcialidad Mbya (Azevedo et al., 2009). Su hábitat tradicional, la ecorregión denominada Selva Paranaense o Bosque Atlántico del Alto Paraná, ha sido reducida a sólo el 7.8\% de su extensión original (Placci \& Di Bitetti, 2006). Los remanentes de la selva misionera que aún subsisten han sufrido profundas alteraciones debidas a la explotación intensiva de los recursos maderables, y sus fronteras se desplazan a diario como consecuencia del avance de los monocultivos forestales y agrícolas, de la urbanización y de otros procesos. Muchas comunidades Mbya son desplazadas y reubicadas en predios que a veces no reúnen las condiciones que tradicionalmente ellos han requerido en cuanto a superficie de suelos cultivables y diversidad de recursos silvestres.

\section{Material y Métodos}

La lista de plantas con fitónimos que denotan la pertenencia a animales ha sido obtenida a lo largo de doce años de investigación etnobotánica en la zona centro y sur de la provincia, en la cual se han entrevistado a no menos de ochenta individuos, de ambos sexos y amplio rango etario, principalmente de la parcialidad Mbya y en menor medida Ava Chiripa, distribuidos en 21 comunidades guaraníes. En dichas campañas se han recolectado las muestras de herbario requeridas para proceder a la identificación de las especies. Los ejemplares originales de dicha colección se hallan depositados en el herbario CTES del Instituto de Botánica del Nordeste, Corrientes, Argentina.

Experiencias previas (Keller, 2003) han demostrado que por más que los interlocutores hablen fluidamente el español, hay aspectos conceptuales y detalles vivenciales que sólo afloran en las narrativas que son registradas en 
la lengua propia de los mismos. Por esta razón los diálogos interculturales, concernientes a la presente investigación han sido registrados en lengua guaraní.

Respecto a la perspectiva teóricoepistemológica empleada, la presente investigación ha debido identificarse con aproximaciones propias de las Ciencias Sociales por cuanto las respuestas obtenidas a lo largo de su desarrollo han contribuido a concebir a la etnobiología guaraní, es decir a la relación de dicha cultura con su entorno biológico, como una interacción social que define a los actores que participan en ella. Como contribución a la etnotaxonomía guaraní, este trabajo constituye sólo una primera aproximación tendiente a sopesar los límites de la aplicabilidad de dicha disciplina.

El método empleado para arribar a los resultados aquí expuestos no responde realmente a procedimientos concebidos $a$ priori, pero visto en retrospectiva es posible reconocer en el desarrollo del presente estudio, los momentos de la dinámica general de la investigación que Guber (2006) identifica en su tratamiento sobre la entrevista etnográfica; una primera etapa que permite al investigador descubrir las preguntas, armando un marco de términos y referencias significativo para las futuras investigaciones y una segunda etapa caracterizada por la búsqueda continua dentro de los nuevos límites fijados en la primera, es decir con una mayor directividad que ayuda a cerrar temas y a ponderar los niveles de generalización de la información obtenida.

El ejercicio de reflexividad desarrollado a lo largo de más de una década de experiencia de investigación permite añadir que gracias a esta dinámica de aproximación paulatinamente concéntrica es posible acorralar a un número sugestivamente mayor de interrogantes significativas. Por otra parte la sostenida consagración a un tópico de investigación lentamente hace palidecer en el investigador la frontera epistemológica entre la teoría y la práctica, de tal manera que la lectura de los antecedentes y la adquisición paulatina de los fundamentos teóricos inherentes al tema de investigación, constituyen asimismo prácticas que no son esencialmente diferentes a la labor pragmática desarrollada en el campo, por cuanto encajan perfectamente en ella, las dos etapas de la dinámica etnográfica referidas por Guber (2006). Una experiencia de campo se puede teorizar, o no, pero los supuestos teóricos por más impresos en papel que se encuentren implican siempre un acto de experimentación, en tanto que sin una vivencia valorativa de los mismos por parte de los lectores, son, a lo sumo, simples manchas de tinta alojadas sobre un sustrato celulósico.

Los interrogantes que cobran vida y se abren paso transitando indistintamente instancias prácticas y teóricas, comienzan a adquirir poco a poco su propio movimiento, y en cierta manera a independizarse del investigador. De este modo, el deterioro de la frontera entre teoría y práctica difumina también el dualismo entre sujeto investigador y objeto de investigación, no discursivamente sino de hecho, ya que la vivencia interpretativa de un fundamento teórico, al igual que la lectura de una "situación de campo", implican un proceso de reflexión especular en el cual el lector es asimismo leído. Es decir, si el texto teórico o la situación pragmática a estudiar no logran interpelarlo, si no interceptan y activan de inmediato o a futuro alguna "fibra sensible" del mismo, no hay lectura, ni lector de los mismos, no hay interpretación, ni intérprete. La emancipación de los interrogantes que adquieren desplazamiento propio constituye un proceso dinámico que difumina al sujeto investigador, le quita relevancia, lo que no responde a presunciones ingenuas de objetividad, ni a estrategias fenomenológicas, más bien implica una disolución empírica de la dialógica estática y categórica entre sujeto investigador y objeto de investigación. Tal es así que la construcción de un marco legítimo de términos y referencias y el descubrimiento de las preguntas significativas para esta investigación han involucrado entrevistas "que llevó a cabo la etnografía" con el investigador con el fin de determinar su disposición para afrontar un determinado problema etnográfico. Las instancias de dicha entrevista han abarcado tanto las experiencias de campo, como la lectura de los antecedentes específicos y teóricos, las dificultades de aplicar las ofertas disponibles de perspectivas analíticas para trabajar la fitonimia guaraní, y el tratamiento etnográfico reflexivo 
de cuestiones colindantes, como constituyentes del escenario mínimo sobre el cual ese asunto de las "plantas de los animales", hasta entonces un tema menor, adquirió movimiento propio como un fascinante enigma.

\section{Resultados y Discusión}

En el apéndice se detallan 188 especies mencionadas por los guaraníes de Misiones como "plantas de los animales", de las cuales 171 ( 91\%) cuentan con registros de uso en procedimientos mágicos. Las plantas listadas distribuyen su pertenencia en 82 animales, de los cuales un poco más de la mitad (45) poseen más de una planta. Los animales con más de cinco plantas son "la garrapata" (10), el ave "urú" o "codorniz de selva" (8), el conejo "tapetí" (7), el venado, el ave "surucuá" y el gallo con 6 especies cada uno. Los fitónimos listados en el apéndice corresponden a plantas que pertenecen a aves (34\%), mamíferos (31\%), artrópodos (16\%), reptiles (13\%) y en menor medida peces (3\%), anfibios (2\%) $\mathrm{y}$ moluscos $(2 \%)$. Cabe reiterar que hay algunas "plantas de animales" a las que no se registraron usos mágicos y aclarar que hay plantas mágicas que no se corresponden con "las plantas de los animales".

A continuación se exponen una serie de argumentos que permiten entender las razones por las cuales para los guaraníes muchas plantas pertenecen a ciertos animales y las razones por las cuales estas son tan frecuentemente usadas en procedimientos mágicos.

\title{
Nuestro antepasado de la primera tierra: el primer taxónomo
}

\begin{abstract}
28/07/2011
H.: -¿Mba'ere katu vicho kuery, teju rami, oguereko ika'a? ¿Mba'echa vypa vicho kuery oguereko, oñemomba'e raka'e?

-¿Porqué los animales, como por ejemplo el lagarto, tienen sus plantas? ¿Cómo es que las tienen, (cómo) se hicieron dueños (de las mismas)?

V.D.: -Néi... ja upe ja echa voiko yvoty, miña ijayu ja'e agỹ reve ja echaramo telepy, dibujo e'irami. Néi, yma upeaja ymave jaiko ẽ̃ tiempo guare, ni juru'a kuery ikua'i e'ỹre guare, ja 'echama voi yvoty урура, ipopa, entonce ijayu joupe. A'erami apyma omombe'u joupe mba'echaguarapa a'ekuery. Néi upea ñande ru voipy ombojera, ñande ru tepy omombe' $u$ : -iNde a'eramiguara, nde a'eramiguara! e'i, e'i aguerami rivepy agỹ "a'e va'e ka' a" ja'e aguã. A'epy ñanderu tenonde gua ja'e porãcheramo ijyvy tenonde va'e; a'e va'era ombojerapa katu iva'ekuepy animal rã.

-Bueno... vemos aquí que (simplemente) hay flores, pero debemos decir que (en verdad) ellas hablan, hasta en la actualidad, hablan del mismo modo que cuando vemos dibujos animados en la televisión. Pues bien, de ese modo (acontecía) antiguamente, muy antiguamente en el tiempo en que no existíamos, ni existían los "barbudos" (persona occidental), podríamos ver a las plantas con pies, con manos; en ese entonces hablaban entre ellas. De ese modo ya se comentaban entre sí (de) quienes ellas eran. Y bueno en esas circunstancias es que nuestro antepasado (de la primera tierra) efectuó las transformaciones, a ellas nuestro antepasado indicó: - $i$ Vos serás tal cosa, vos serás tal otra!- indicaba; simplemente por haber dicho eso es que hasta ahora podemos decir que son "las plantas de ellos" (de los animales). Así que fue aquel antepasado nuestro, al que llamamos el de la primera tierra, fue él quien transformó (a aquellos hombres) en las cosas que serían para los animales.
\end{abstract}

Puede notarse que esta respuesta remite a acontecimientos de índole cosmogónica, destacándose la naturaleza eminentemente humana del creador (nuestro ancestro de la primera tierra), así como también de la materia prima sobre la cual dicho antepasado primigenio diseñó a la mayoría de las plantas y animales. En cierto modo la expresión ¡serás tal cosa!, permite calificar a este primer antepasado como el primer taxónomo guaraní. Según Bartolomé (2009) para los Mbya el nombre y lo nombrado son datos 
irresolublemente ligados en la conciencia del hablante, la palabra participa de las propiedades de lo que nombra. De modo que se podría decir que fue dicho personaje quien concibió las primeras plantas y animales y plasmó dicha labor taxonómica original en el equivalente guaraní de los "ejemplares tipo", es decir la descripción de cada especie es nada menos que el mismo ejemplar primigenio, obra de su creatividad a partir de rasgos sugerentes que le inspiraban ciertos facciones o actitudes, generalmente reprobables, de especímenes humanos. La literatura sobre los guaraníes ofrece numerosos ejemplos de estas transformaciones de sujetos que han faltado a ciertas normas de conducta culturalmente establecidas (Cebolla Badie, 2000; Keller, 2003, 2007b, 2009, entre otros). Se transcribe a continuación un registro sobre este tipo de transformaciones, el cual ofrece argumentos para entender porqué la "hierba del eirachĩ" se utiliza en procedimientos de magia destinados a enfermar a los adversarios de disputas amorosas. Se trata de la creación del dueño de esta planta, el melipónido Lestremelitta limao, denominado "eirachĩ" (miel que enferma) o "yvyra'ija" (dueño de los troncos), y de cuyas colmenas los guaraníes extraen miel.

\section{$14 / 08 / 2011$}

C.B. -Eirachĩ oiko raka'e ojapychaka opy, oiko guvicha. A'upéi naimborayui, imbavyky, ojapo opa mba'e; oiporu tatachirã py, gui katu oiko ñombojecha vai oikovy; ojapo pindo ro'ykãguy, mba'ekuaa, ojapo ita rayi gui mba' 'kuaa, ojapo avi yvyra ta'y rakua gui ojapo avi, tonche ojukapa kyryngue 'ipe, kyrynguepe 'ỹ ave. A'erami ramo a 'epy ojavypy guvichape, ñanderurãpe; a' eramivy uvicha ombojera raka'e eirachĩ ipy, eirachĩrã raka'e ombojera raka'e. A'erire ko angã pe ve eirachĩ, naimborayu'i anuã rami, ndopotái ijyy iry ñavaẽmavy ñande ayu, jacha rive ñakyrirĩ va'era, ñañande ayu'ira, ñande ayuramo ñaneñyvo; a'erami e'ỹramo ñande mbopire raku rive. Nde pire raku ma ramo o chino ñeyvo ma ramo re'orapy opygua apy ndepychy ãnuã, ñemongeraju ãnuã. A'eramivyje eirachĩ reitymavy nda'eira Vera, Vera ery va'e ndojo 'oira, Karai ery va'e ojo'orã, oguenõ ãnuã ei. Tonche ojo 'otamavy yjayurapy okére oinupa-nupa rapy achapy a'upéi: -jEñongatupa ke nderu'y!- e'irã. A'eramiramo oñongatupa gu'y a'erami javepy omboguáirã oguenõ ãnuã ei.

-Existía quien (se llamaba) Eirachĩ, escuchaba en el templo, ejercía su liderazgo. Pero no era generoso, practicaba la hechicería, hacía de todo; usaba la niebla sagrada, pero también solía practicar maleficios; confeccionaba (dardos shamánicos) con la vena media de la hoja de la palmera pindó, (aplicaba esa) sabiduría maligna; confeccionaba bolitas de roca (aplicaba esa) sabiduría maligna (las introducía en los cuerpos); confeccionaba también dardos de la madera de alecrín (Holocalyx balansae: Fabaceae); entonces asesinaba a los niños y también a los no tan niños (adultos). Siendo así se desvió de los preceptos del líder, de nuestro futuro ancestro; entonces este lo transformó en el eirachĩ primigenio, en el futuro eirachĩ lo transformó. Después de ello, hasta el presente, el eirachĩ es igualmente mezquino, del mismo modo tampoco admite que hablemos al aproximarnos (al árbol que habita), aunque simplemente debamos pasar (cerca de su colmena) nos callamos, si decimos apenas alguna cosa, al hablar ya nos arroja sus dardos, de no ser así simplemente nos da fiebre. Cuando te da fiebre o te arroja los dardos debes ir con el sacerdote a fin de que te fumigue para que puedas curarte. El tema es que para recolectar la miel de eirachĩ no debes ser Vera, quien porte el patronímico Vera no puede ahuecar (el tronco), quien porte el patronímico Karai deberá excavarlo, y podrá extraer la miel. Entonces habiéndolo ahuecado, en la entrada del panal, golpeando reiteradamente con su hacha, debe exclamar: -Oculta tus dardos! - dirá. De ese modo cuando (Eirachĩ) oculte (sus dardos) ahí recién procederá a cortar (el nido) para extraer la miel.

Es interesante denotar que la noción de que la humanidad constituye la materia prima sobre el cual se crearon y aún se pueden crear plantas y animales, además de conformar un sustrato propicio para el establecimiento y la transmisión de normas de conducta (ej: ¡no practicarás la hechicería maligna!), sólo admite la posibilidad de "utilización de recursos naturales" como un acto de interacción 
social. -¡Enfunda tus dardos!, ordena el recolector antes de proceder a la destruir el nido de su interlocutor melipónido, para efectuar la extracción de la miel. Estas interacciones adhieren al postulado que expresa Bartolomé (2009) al trabajar sobre el discurso cosmológico guaraní: la indiferenciación primigenia es la que establece la ambigüedad o permeabilidad de la distinción ontológica entre naturaleza $y$ cultura. Esta perspectiva es además sugestiva por cuanto, desde ella, la ingestión de animales y plantas no puede eludir cierta filiación con el canibalismo ${ }^{1}$, ofreciendo una alternativa de interpretación cultural menos demonizante que la blandida históricamente para comprender la antropofagia de los amerindios $^{2}$, documentada también para los guaraníes agricultores (Fausto, 2005; Metraux, 1948).

\title{
El rival de nuestro primer antepasado: el segundo taxónomo (narración mbya)
}

\begin{abstract}
28/07/2011
V.D.: -Ñande ru mbojera ka'a, ka'a'i peicha jaiporu vaerã ñande a'e. Ombojeraramo, Charĩa katu ombojera ka'avomapy. Ei miña a'erami avi; eiropa ombojera (ñande ru) rangaramo ombojeraju Charĩa mamanga ju, jatei ju ombojeraramo a'e ombojeraju kavyju, narã ju ombojeraramo a'e ombojeraju apepu, pindo ju ombojeramo a'e ombojeraju mbokaja...

-Nuestro padre creó las plantas que serían de nuestra utilidad. Al crearlas, (tratando de imitarlo) su rival creó las plantas para hechizar. Lo mismo ocurrió con la miel, cuando (nuestro padre) creó la abeja, su rival creó al mamangá (una abeja ponzoñosa que no produce miel), cuando nuestro padre creó al yatei (melipónido que produce miel medicinal), él creó la avispa carnicera, cuando (nuestro padre) creo a la naranja, él creó al apepú (naranja agria), cuando (nuestro padre) creó la palmera pindo ${ }^{3}$, él creó al mbokaja (palmera espinosa no apta para la construcción)...
\end{abstract}

Así como hay plantas y animales que resultan de utilidad para los guaraníes, hay otras que además de inservibles pueden resultar desagradables o dañinas. Se trata de las creaciones de Charĩã, el rival del primer antepasado guaraní, quien tratando de emular los prototipos de este, creó imitaciones "de mala calidad".

Las plantas útiles son transformaciones efectuadas por el primer antepasado guaraní para servir como recurso a los primeros habitantes y a las generaciones venideras. Estas transformaciones, así como las del rival mencionado, se efectuaron sobre la base de ciertos rasgos sugerentes de quienes fueron transformados. La inspiración creativa se conformó a partir de dichos rasgos, lo cual no es un detalle menor, puesto que esta dinámica de diferenciación biológica prefigura las condiciones de aplicación de la doctrina de la signatura para la selección de especies útiles, principalmente las empleadas por los guaraníes en medicina tradicional y magia; esto implica que el uso específico de muchas especies se halla determinado a partir de ciertos rasgos sugerentes; por ejemplo plantas con espinas son usadas para tratar odontalgias, o bien plantas frías al tacto son usadas para bajar la fiebre, por mencionar sólo algunas vinculaciones (cfr. Keller, 2007a). Es decir, si el creador y su rival diseñaron sus obras vegetales a partir de ciertos rasgos especiales

${ }^{1}$ Como lo expresa Viveiros de Castro (1996) al abordar el canibalismo amerindio: "la semejanza de los espíritus prevalece sobre las diferencias de los cuerpos".

${ }^{2} \mathrm{La}$ antropofagia amerindia fue uno de los primeros neologismos americanos y uno de los más recurrentes descalificativos que la sociedad europea de "los grandes descubrimientos" manejó para definir la alteridad transoceánica (Mora, 2008).

${ }^{3}$ En este punto de la narración R.D. intercedió explicando que la palmera pindó primigenia fue creada por el primer ancestro, quien tras requerir material para cubrir el techo de su vivienda observó a una mujer con voluminosa cabellera, a quien transformó en palmera para usar sus cabellos devenidos en hojas. 
de quienes serían transformados, entonces quienes deseen utilizar dichas creaciones en su beneficio deben buscar la prescripción para atender un determinado problema en los rasgos especiales que presentan las plantas.

Los intentos de Charĩa por emular los actos creativos del primer ancestro, su rival, entre otras cosas, resultaron en la creación, también a partir de humanos, de las plantas que serían empleadas para hechizar. La expresión "ka'avo" es el apelativo más usual con que los guaraníes designan a las plantas mágicas (Keller, 2007a), también se aplican a las yerbas nocivas introducidas en las vísceras del embrujado (Cadogan,
1992). Sugestivamente, en el idioma guaraní clásico la expresión se puede traducir como planta cultivada, hortaliza o verdura (Guasch \& Ortiz, 1995) y el término puede descomponerse en $k a^{\prime} a=$ planta y $\tilde{o}=$ hoja, es decir "planta de hoja" o "planta en la cual la hoja es lo más relevante". Si quienes practicaban la hechicería u obraban mal serían transformados en animales, entonces sus hortalizas, las plantas que cultivaban siendo personas (sus antiguos asistentes), son aquellas que hoy son conocidas como "las plantas de los animales", se trata mayormente de plantas usadas en hechicería, creaciones del rival del primer guaraní.

\section{8/05/2011}

R.D.: -Ymave raka'e pavẽ vichorã ijypy raka'e, aupei ma'ẽ oñembojerama vicho. Teju ypy oguereko raka'e guemiñoty ae' $i$, imbae ae. A'egui ae ojavy ñanderupe, ñande ru ombojera tejurã. A'upéi teju ypy omano ramo iñe'ẽ opyta emiñoty kuere; a'eramivypy jaikua'a ãguã teju ka'a.

-Antiguamente todos los animales primigenios eran (gente), y recién después fueron convertidos a animales. (Por ejemplo la persona que sería transformada en) el lagarto primigenio tenía sus (plantas) cultivadas, les pertenecían. Entonces se desvió (de los preceptos) de nuestro antepasado, y este lo transformó en lo que sería un lagarto. Luego, cuando ese lagarto primigenio murió su alma permaneció en las plantas que cultivaba, de ese modo es como concebimos a la hierba del lagarto.

\section{La esquiva noción de propiedad privada 0 pertenencia}

Entre los rasgos sugerentes que denotan a qué animal pertenece una determinada planta, se encuentran algunos del tipo morfológico, por ejemplo la "hierba de la serpiente" presenta flores que se asemejan a ofidios (Fig. 1A); mientras que la "hierba del pato" presenta flores similares a esta ave; las flores de la "hierba del mono aullador" (Fig. 1B) son morfológicamente similares al hueso hioides de este mamífero el cual le sirve para emitir sus aullidos; las inflorescencias de la "hierba del gallo" (Fig. 1C) se asemejan a la cola de dicha ave doméstica (Fig. 1D): los zarcillos de la "planta del jaguar" (Fig. 1E) se parecen a garras felinas. Otros rasgos sugerentes tienen que ver con el aroma, por ejemplo la mencionada hierba del "eirachĩ " presenta un aroma similar al que despide el melipónido en cuestión. También los guaraníes tienen en consideración aspectos etnoecológicos, tal como sugiere Bertoni (op. cit.) es decir reconocen el hecho de que muchas plantas de los animales crecen en los ambientes donde estos frecuentan, así por ejemplo es usual hallar a las plantas "de la rana", las plantas "del pez" y las plantas "de la tortuga" (Fig. 1F) en ambientes acuáticos; en cambio las "plantas del toro" son abundantes en los campos de pastoreo (Fig. 1G). Como se puede leer a continuación, la tradición oral guaraní aún conserva buenas explicaciones para abordar estas regularidades etnoecológicas.

28/07/2011

R.D.: - ¿Ña'a mba'ere katu kururu..., mba'eiko a veche ko kururu ka'a etãpy oikora reima kururu, chino katu churuku'a ka'a etãpy churuku'a ovy'ara reima?

-¿Pero por qué razón por ejemplo el sapo...?.¿Cómo es que a veces donde abundan las "plantas del sapo" es probable encontrar al sapo, o bien donde abunda la "planta del surucuá", el surucuá se encuentra a gusto?. 

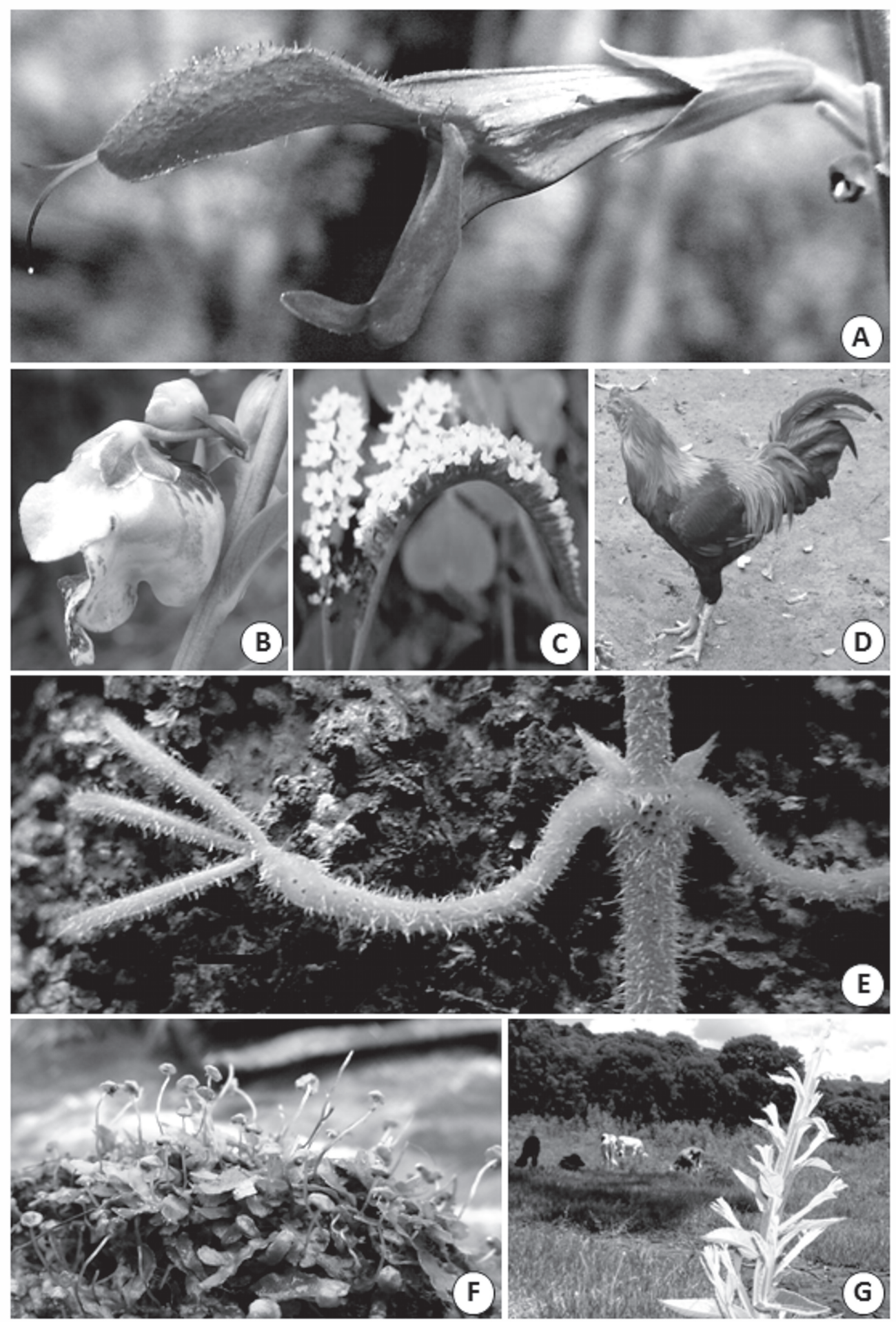

Fig. 1. A: Flor de Salvia guaranitica (Lamiaceae), una de las hierbas de la serpiente. B: Flor de Angelonia integerrima (Scrophulariaceae) una del las plantas del mono aullador. C: Inflorescencias de Heliotropium transalpinum (Boraginaceae), una de las plantas del gallo. D: Ejemplar de gallo. E: Zarcillo de Dolichandra uncata (Bignoniaceae), una de las plantas del jaguar. F: La planta de la tortuga (Marchantiaceae), creciendo en un ambiente acuático. G: Ejemplar de Pterocaulon balansae (Asteraceae), una de las plantas del toro, creciendo en un campo de pastoreo. 
H. A. Keller, Problemas de la etnotaxonomía guaraní: "Las plantas de los animales"

V.D.: -Ja okua'apy oka'a, oka'are oñatende avipy. Néi agỹ miña oĩ ka'a; chino chee, chino ndee rereko ka'aty... jurua kuery mba'e a'erami imba'erima, ja'e porãcheramo: "iplanta", "a'ekuery $k a$ 'a" ja'e,... ¿mba'eichapa ja'erã?: ochẽ a'ekuery akãte'y ama, akãte'yramo rivepy: "ika'a" ja'e. Ngau che areko peteĩ ka'aty, entonche upea ja che ka'amapy, chee cherakãte'ypy che plantare a'eramipy o'oiny. Upe ika'a ja'e aguã va'e katu a'ekuery, yvoty memema o chino planta rivemapy oñemombe'u: -;Chee, a'eva'erima cheja!-, amba'emapy ikuái, yma ymave ijayupy, agỹ reveranjepe ijayu, pero ndajaikua'aipy. Ni cualquier animal ipy ijayu ojoupe, pero ñande kuery ndaja'echái, ñanaenduvéimapy ñomongetaramo, mba'epa e'i a'ekuery joecha. Peicha ngau, peicha kapi'i-pi'i joyvy-yvy ikuái; lo mimo a'ekuery ipueblopy ikuái, a'eramivy ñomongeta, a'eramivypy omombe'u joupe mba'e-mba'epa a'e. A'eramivypy jaikuaa aguã churuku'a ka'a, kururu ka'a ja'e aguã.

-(Es porqué esos animales) conocen sus plantas, y se encuentran cuidándolas. Bueno, pero hasta hoy en día hay plantas (así); supongamos que yo o bien vos tenés una plantación... con los "barbudos" (persona occidental) ocurre lo mismo, son cosas de su pertenencia, si lo expresamos correctamente son "sus plantas", "las plantas suyas" decimos... ¿cómo podríamos expresarlo?...eso surge a causa de que ellos protegen (sus plantas), y simplemente por protegerlas es que usamos la expresión "su planta". Supongamos que yo tenga un yerbal, entonces pues ese es ya "mi yerbal", yo protejo mis plantas, y así sucesivamente. Decimos "su planta" al referirnos a cosas que son de su pertenencia, y de ese modo todas esas flores (señala un jardín), o simplemente las plantas, se comentan entre sí: -¡Heme aquí, aquel es mi dueño!, -todas ellas son así, antes, antiguamente hablaban, y hasta ahora hablan, pero no lo comprendemos. Asimismo los animales, cualquiera sean, en principio hablan entre sí, pero nosotros no lo percibimos, no podemos escucharlos mientras conversan, (y saber) qué cosas se dicen los unos a los otros. Imaginemos entonces a los pastos (señala el césped) que se encuentran muy juntos, uno al lado del otro, es como si estuvieran en su pueblo, y así es como conversan, así es como se cuentan entre ellos como son las cosas. Así es como concebimos a las plantas del surucuá, a las plantas que decimos que son del sapo (etc.).

La primera parte de la narración pone en evidencia dificultades en definir que algunas plantas o animales, otrora personas, puedan ser propietarias, o estar sujetas a la propiedad privada. La avenida más propicia para una factible interpretación desde la óptica del interlocutor mbya es considerar el acto de apropiación como sinónimo de apadrinamiento o custodia, del mismo modo en que se protege a niños huérfanos o a un séquito de personas a cargo; que usualmente devienen en sirvientes o emisarios (R.D. com. pers.).

Lasegundaparte delanarración sugiere cierta filiación con postulados del perspectivismo amerindio (cfr. Viveiros de Castro, 1992) en tanto que las especies animales y vegetales aparecen como si fueran meros envoltorios que esconden una forma interna humana, normalmente visible apenas a los ojos de la propia especie.

La dotación del mismo nombre a más de una planta, particularmente de plantas de los animales, muchas veces genera incertidumbre en quien registra fitónimos guaraníes, sin embargo el siguiente diálogo ofrece una buena explicación para tratar ese asunto.

\section{$28 / 05 / 2011$}

H.: - ¿Õvepa ka'avo peteĩ teĩ vichoguy?

- ¿Hay más de una planta de cada animal?

R.D.: -Oñve ioñembojera va'ekue oñoty reta ramo oka'avorã

-Las hay, si la persona que fue convertida cultivó muchas plantas

H.: -¿Pavẽ pa peteĩ vichore ka'a aeramingua rive, peteĩ kuña no'erayu aguã rive?

- ¿Todas las plantas de un animal son igualmente efectivas, por ejemplo para enamorar a una mujer? 
R.D.: -Õ̃ avi peteĩ nguy aeve ve va'e, eãkuã rachyve va'e. Orekuery rami oguereko oipotave va'e, a'erami avi vicho ka'a. Ojeporuve va'e eãkuã ve, imbareteve a'e vaepe roeñói "ka'avo pyta'i”, teju ka'a pyta'i. Na eãkuãive va'e pe katu roeñói "ka'avo jaryi".

-Hay algunas que son mejores, las de aroma fuerte. Hasta nosotros tenemos algunos (cultivos) que son los preferidos, lo mismo ocurre con las plantas de los animales. Las más efectivas son las más aromáticas, las más fuertes; a esas nosotros las llamamos "plantas mágicas concentradas", (por ejemplo) "planta del lagarto concentrada". A las que no tienen tanto aroma las llamamos "plantas mágicas débiles"

Se considera que algunas de las plantas que pertenecen al mismo animal son más concentradas que otras, en su fragancia y en su poder, debido a que han sido las plantas más apreciadas por quien en vida las cultivaba, lo cual implica que una mayor fracción de su alma telúrica reside en ellas ${ }^{1}$. Las menos apreciadas pueden no tener fragancia, o esta es muy leve y no se esperan resultados tan promisorios al usarlas en procedimientos mágicos.

\section{La propiedad privada, un problema de ultratumba}

El apremio por poseer un bien material, como una planta, implica para los guaraníes un problema que recién se agrava con la muerte del propietario, pues ellos consideran que parte de la voluntad de cada persona, particularmente la que expresa ansiedad por sus pertenencias, permanece en la tierra fuertemente ligada a las mismas. Mientras que el alma dotada por los dioses retorna a su morada celestial, el alma telúrica, que denominan "angue" (sombra) ó "ñe'ẽ vai" (alma mala), o "teko achy kue" (resultado de las imperfecciones) deviene en un fantasma muy temido al que denominan "mbogua". Dicho fantasma merodea los alrededores de la que fuera su morada terrenal causando pánico y enfermedades, practicando asimismo la hechicería maligna. Por esta razón los cementerios guaraníes generalmente se hallan alejados de las aldeas y el muerto es enterrado sentado de cuclillas o acostado dentro de una cesta de bambú, que emula el vientre materno. Además, con el fin de mantener subsumido en sus pertenencias al fantasma en cuestión, se colocan sobre la tumba los elementos que en vida más apreciaba o frecuentaba, tales como herramientas, utensilios de cocina, armas, semillas, bebidas alcohólicas y otros (Fig. 2A y B). Inclusive sobre las tumbas de los niños lactantes usualmente colocan recipientes con leche para que el fantasma no inquiete a la madre del párvulo difunto (R.D. com. pers.). Además, en los días más fríos del invierno se suelen encender fogatas cercas de las tumbas, para que los fantasmas no merodeen las viviendas en busca del calor de los hogares (J.C.B., com. pers.).

Los guaraníes desarrollaron una notable agricultura desde hace más de 2000 años (Schmitz, 1991), constituyéndose hasta la actualidad en una de sus principales estrategias de subsistencia. Los cultivos ocupan, por lo tanto, un lugar privilegiado entre los bienes de los integrantes de las comunidades, vale decir, locus o sitios propicios para el alojamiento de los fantasmas mencionados.

\section{8/05/2011}

H.: -Peteĩngue remombeu karamboae pave ñaneñe'ẽ mokõi'i va'e, teju rami ¿mba'echagua ñe'ẽ katu opyta ika'avore?

-En una oportunidad me comentaste que cada persona tiene dos almas, (supongamos) en el caso del lagarto (primigenio) ¿Cuál alma quedó en la planta?

${ }^{1}$ Ciertas correspondencias entre la noción guaraní de "alma telúrica" y los efluvios aromáticos fueron sugeridas por Keller (2007a) para explicar los recaudos corporales que toman los guaraníes para evitar ser percibidos por predadores naturales y anímicos. 
R.D.: -Iñe'ẽ vaikue, pave vicho ndoguerekoi amboa'e

-El alma telúrica, los animales no tienen otra

H.: -A'emavy peteĩ ava oiporuramo teju ka'a peteĩ kuña oayu aguã ¿Mba'echa katu omba apo teju $k a ' a$ ?

-Entonces en el caso de que un hombre utilice la planta del lagarto para enamorar a una mujer ¿Cómo es que trabaja esa planta?

R.D.: -Ndee reikua'a teju ka'a, reikua'ama eãkuã rachy, pavẽ vicho ka'a eãkuã. Teju ñe'ẽ rive aetu eãkuã va'e, eãkuã va'e rivema ombaapo, omyĩ yvaterupi eãkuãngue. Omỹ̃vy a'epy ojapo ñaĩkoteve va'e, ojapo peteĩ kuña oayu aguã peteĩ avape.

-Vos conocés la planta del lagarto, ya sabés que tiene olor fuerte, todas las plantas de los animales tienen aroma. El alma del lagarto es ese aroma, mediante ese olor trabaja, puede desplazarse por el aire mediante esa fragancia. (Entonces) si puede moverse, puede hacer el trabajo que le piden, puede (por ejemplo) hacer que una mujer se enamore de un hombre.

H.: -¿Mba'epa opygua kuery oicha' ã ka'avo oiporu va'ere?

-¿Qué opinan los líderes espirituales acerca de los que utilizan plantas mágicas?

R.D.: -Pavẽ opygua kuery ndo ikuaachéi, mba'eiko vicho ñe'ẽ rivepy, aeva'e ndoipotái, ñande ru kuery mba'e'ỹi py aeva'e

-Los sacerdotes no quieren saber nada del asunto, porque se trata del alma de los animales, eso no les agrada, no son asuntos de los dioses

Las especies vegetales silvestres que entrañan "los cultivos de quienes fueran transformados en animales", son dispositivos cargados con el poder de sus antiguos cultores, cuyas "almas telúricas" se hallan subsumidas en el aroma de dichas plantas.

De este modo, las plantas mágicas "que pertenecen a los animales" constituyen el modo más frecuente para atender apremios amorosos, es una vía accesible para vincularse transactivamente con el "alma telúrica" de sujetos transformados en animales primigenios y obtener resultados rápidos a bajo costo. Dichos espíritus siempre se hallan con ansias de involucrarse en apremios "terrenales", razón por la cual sus ofertas son asequibles. Entre los modos más frecuentes de saldar las cuentas con ellos y retribuir los favores recibidos se destacan compromisos triviales como por ejemplo devolver las hojas extraídas al lugar de hallazgo, desmalezar el sitio donde la planta usada crece y velar por su cuidado. Los pedidos y los compromisos pactados se deben expresar en voz alta. Ante el incumplimiento de pactos tan lábiles, la máxima venganza posible que puede tomarse el "espíritu animal" contra quien lo "ha contratado", consiste en desarticular el hechizo, disolviendo el encanto o enamoramiento logrado, lo cual, muchas veces, una vez consumada la intención, no representa inconveniente alguno para los usuarios que buscan satisfacciones meramente carnales. Pero un "hechizo" mal consumado, puede en cambio afectar la salud del destinatario o destinataria, en cuyo caso el referente religioso de la aldea, haciendo uso del poder que le confieren los dioses, debe extraer el paquete de hojas usado en el embrujo amoroso (Fig. 2C), que usualmente se encuentra alojado en la cabeza de la víctima (R.D. com. pers.).

\section{¿Plantas de plantas?}

La perspectiva cosmogónica expuesta admite la existencia de numerosas "plantas de los animales", y si se tiene en cuenta la condición humana primigenia de las especies involucradas, es de suponer que los guaraníes también podrían llegar a concebir ciertas "plantas de las plantas"; pues lo hacen.

Se citan en el apéndice dos representantes del género Phoradendron Nutt. (Viscaceae); los cuales son especies hemiparásitas que viven a expensas de hospedantes generalmente 


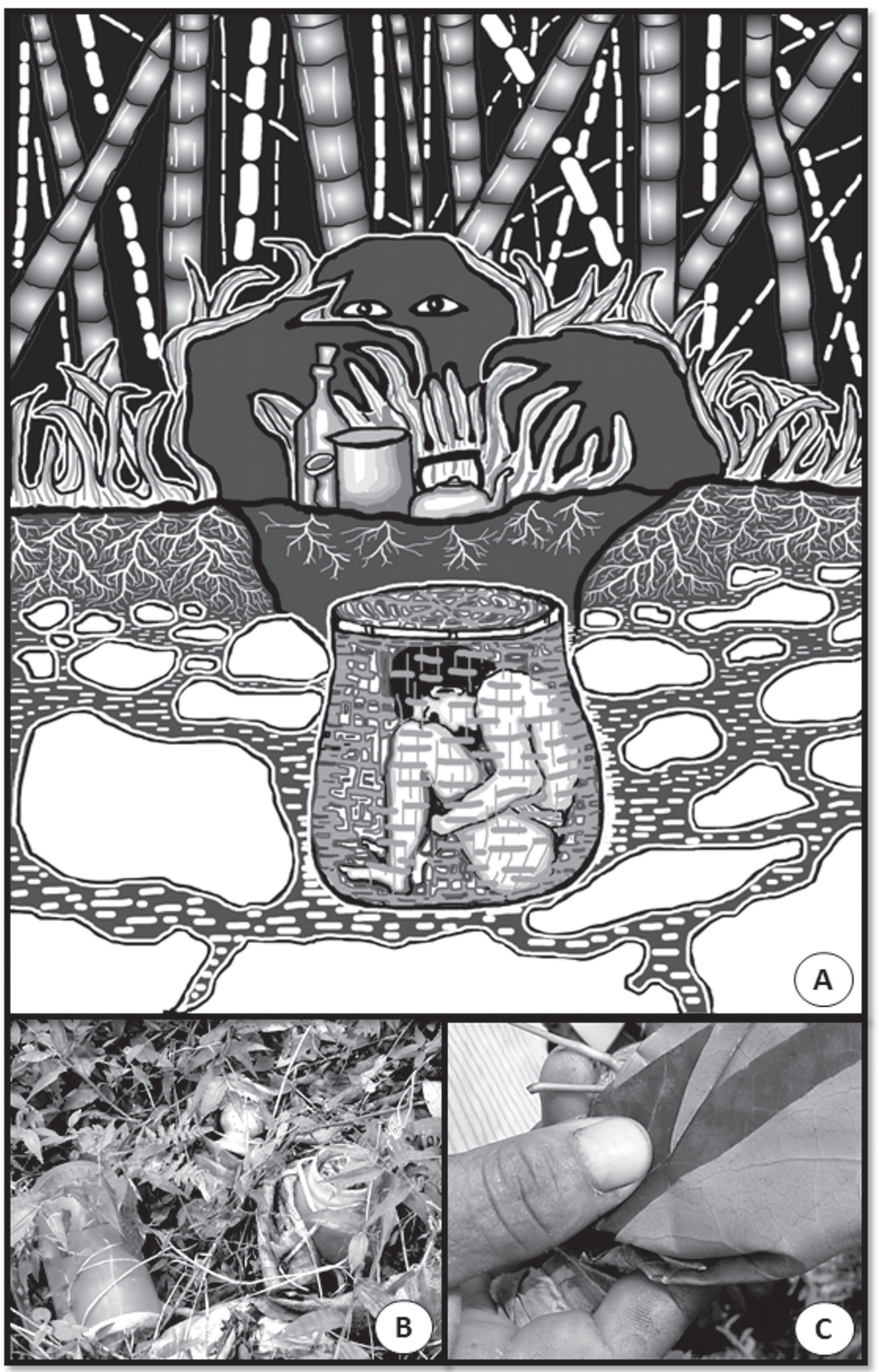

Fig. 2. A: Representación alegórica de un entierro guaraní y del fantasma recluido en las pertenencias del difunto, confeccionada sobre la base de la descripción efectuada por un interlocutor Mbya guaraní (R.). B: Objetos depositados sobre una tumba Mbya guaraní. C: Paquete de hojas usadas en un procedimientos de magia amorosa. 
arbóreos. Sus bases se hallan notoriamente soldadas a las ramas de diversas especies de árboles, por lo que reciben el nombre vernacular de "ligas" y se usan en medicina popular para tratar las fracturas óseas. En la zona de unión o soldadura, el huésped desarrolla raíces haustoriales que le permiten absorber agua y sales minerales desde el xilema de su hospedante; asimismo su posición en lo alto de los árboles le permiten acceder a condiciones de iluminación necesarias para transformar dichos nutrientes en recursos alimenticios. Estas plantas son denominadas "ypeku ka'a" (la planta del pájaro carpintero) y "karaja ka' a" (la planta del mono aullador) por los Mbya guaraní, pero en el dialecto Ava Chiripa son denominadas "ka'avo tyre' 'y". La expresión "tyre' $\ddot{y}$ " se traduce como "huérfano" o "carente de madre" (ty/chy: madre; re' $\tilde{y} / e^{e} \tilde{y}$ : sufijo de negación). En su condición humana primigenia estas especies eran niños huérfanos que fueron adoptados por diversos tutores, los que fueron luego transformados en árboles (C.B. com. pers). En este tipo de relación trófica, la noción de "adopción de un huérfano" en la conformación del fitónimo es interesante porque ofrece una alternativa conceptual y ética a la noción científica de "parasitismo"; la primera entraña el concepto de reciprocidad en tanto que esta última denota privatización y mezquindad de los nutrientes obtenidos del suelo por parte del árbol, y hurto por parte del huésped. Al parecer son explicaciones disímiles de un mismo fenómeno biológico, elaboradas a partir de modelos idiosincráticos diferentes. Puesto que es absurdo testear científicamente la buena o mala voluntad con la que cada árbol hospedante destina un sitio iluminado entre sus ramas y obsequia parte de sus "víveres" a otra planta más pequeña; ambas propuestas deberán permanecer a nivel conjetural hasta tanto no volvamos a comprender "lo que las plantas se dicen las unas a las otras".

\section{Reflexiones finales}

La Etnobiología ha padecido los ataques dirigidos contralos esfuerzos etnotaxonómicos, principalmente los de perfil universalista, por su insistencia en enfatizar los niveles de convergencia entre la clasificación folk y la clasificación biológica, insistencia que es vista como un intento de evaluar el saber indígena en términos de su compatibilidad con la ciencia occidental (Hviding, 2001). Una de las premisas privativas más endebles de la etnotaxonomía universalista es la de considerar que en la idiosincrasia de todos los pueblos, los organismos vivos son dables a ser objetivados y clasificados sistemáticamente como si pertenecieran a un mundo separado de la sociedad humana. Sin duda, sobre este presupuesto Bertoni (1940) imaginaba las asambleas taxonómicas de los guaraníes, en las cuales los caracteres más adecuados para conformar los nombres de plantas se escogían democráticamente.

Inmersos en una perspectiva desde la cual únicamente podemos concebir a la naturaleza como un bien de consumo en cualquiera de sus formas (económico, recreativo, ambiental o científico) es muy difícil concebir la posibilidad de que en ciertas culturas se establezcan relaciones sociales entre humanos, plantas y animales. Sin embargo, cuando un mbya guaraní recoge y guarda entre sus prendas una hoja fragante de "teju ka'a", (la hierba del lagarto) con el fin de enamorar a la dama de sus sueños, no considera simplemente la posibilidad de recurrir a las propiedades químicas de dicha planta, es decir a un perfume que podría tornarlo más atractivo, como sugiere Keller (2010), sino que desde su perspectiva establece una relación social, un vínculo transactivo con el "alma telúrica" de una persona, la cual, por haber sido corruptible ante los apremios terrenales, fue alguna vez transformada en el lagarto primigenio. Desde la muerte física de dicho espécimen de reptil, la esencia telúrica de aquel ser humano condenado reside subsumida en algunas de las hierbas predilectas que antes cultivaba en su huerto y que ahora crecen espontáneamente en los sitios donde frecuentan los lagartos (descendientes de aquel). Las hojas, estrujadas y manipuladas por el practicante de la magia, desprenden un perfume que, en tanto sustancia volátil, vivifica a dicho espíritu telúrico, admite su pervivencia en el reino de lo animado, permitiéndole manifestarse, desplazarse de 
un sitio a otro, trabajar por encargo. Por estas razones la planta en cuestión es conocida como una de las "hierbas del lagarto", y, aunque sea clasificada sin vacilaciones por cualquier mbya como un vegetal silvestre, para él también es, sin contradicción alguna, una planta cultivada, un animal, un espíritu y un ser humano.

Otro ejemplo interesante para esta discusión es el apelativo "manji ka'a" (la hierba de la lagartija), con que los mbya denominan al mantillo de micelios fluorescentes de ciertas especies de hongos. El vocablo " $k a$ ' $a$ " (hierbas) aplicado a hongos luminiscentes fue también citado como un hecho curioso en una obra sobre etnobotánica de los Ka'apor, un grupo Tupi Guaraní amazónico (Balée, 1994). La expresión "manji ka'a" contiene sufijado un clasificador nominal ( $k a^{\prime} a=$ hierba) que determina la "forma de vida" de la especie en cuestión, de modo que mediante los procedimientos etnotaxonómicos usuales, aplicados a una lista cruda de fitónimos guaraníes, se podría dictaminar su membrecía émica entre las "hierbas". Pero una simple entrevista de verificación ulterior no tardaría en quebrantar dicho vaticinio; la escurridiza taxonomía mbya esgrime también el vocablo "kya" (hamaca o tela), con el cual se alude genéricamente al mantillo de micelios que se forma bajo la hojarasca del suelo selvático. Desde un punto de vista simplemente ontológico dicha categoría excluye a las hierbas, es decir que los guaraníes disponen de recursos clasificatorios tan firmes como para perturbar la primer conjetura etnotaxónomica y afirmar que, pese a su clasificador nominal, "la hierba de la lagartija" no es una planta, es simplemente " $k y a$ " (mantillo fúngico). Esta demostración de solidez taxonómica bastaría para compensar la crisis inicial causada por una supuesta nomenclatura endeble, podría aplacar las ansias ontológicas del paradigma taxonómico, es decir su anhelo por aprisionar "lo que es" excluyendo "lo que no es". Pero bajo la fachada rectilínea que ofrece una dimensión clasificatoria simplemente ontológica subyacen dimensiones mitológicas y cosmogónicas, desde las cuales dicho "mantillo de micelios fúngicos" puede ser fehacientemente concebido por un mbya como un hongo, una planta, un animal, una persona, un espíritu. Semejante flexibilidad clasificatoria nos podría insinuar que a pesar de los desvelos etnotaxonómicos de Bertoni (1940), los guaraníes denotan una taxonomía ambigua, o que, como concluyen Newmaster et al. (2006), los pueblos tradicionales sólo identifican organismos, sin clasificarlos; pero también, si nos lo permitimos, podemos atisbar en dicha flexibilidad, las ambigüedades de la taxonomía biológica cuando se la intenta extender más allá del paradigma cultural en el que se halla contenida.

\section{Agradecimientos}

A Vera Mirĩ (R.D.), Kuaray Tupã (V.D.), Vera Tataendy (C.B.) y Vera Chunu (J.C.B.) por la franqueza y paciencia manifestada ante mis inquietudes. A Miguel Bartolomé por orientar y reorientar mi perspectiva etnográfica. A Diana Milstein, Fabián E. Gatti y Rodrigo M. Dutra por la lectura crítica del manuscrito. A Antonio Krapovickas, Gustavo Scarpa, Daphne Colcombet y Ernesto Maeder por la bibliografía suministrada. A los especialistas del Instituto de Botánica del Nordeste por colaborar con la identificación de las plantas. Al Consejo Nacional de Investigaciones Científicas y Tecnológicas por sustentar mis investigaciones.

\section{Bibliografía}

ALONSO, J. \& C. DESMARCHELIER. 2005. Plantas medicinales autóctonas de la Argentina. Bases científicas para su aplicación en atención primaria de la salud. Ed. L.O.L.A. Buenos Aires, 663 pp.

AMAT, A. G. \& M. E. YAJÍA. 1998. Plantas populares utilizadas en la fitoterapia popular de la provincia de Misiones (Argentina). En: A. G. Amat (Coordinador): Farmacobotánica y Farmacognosia en Argentina, 1980-1998. p. 119-152. Ediciones Científicas Americanas. La Plata.

ARENAS, P. \& J. A. BRAUNSTEIN. 1981. Plantas y animales empleados en paquetes y otras formas de la magia amorosa entre los Tobas Tsaksik. Parodiana 1(1): 149-169.

ASSIS, V. \& I. GARLET. 2004. Análise sobre as populações guarani contemporâneas: demografia, espacialidade e questões fundiárias. Revista de Indias 64(230): 35-54. 
ATRAN, S., S. MEDIN \& N. ROSS. 2004. Evolution and devolution of knowledge: a tale of two biologies. Journal of the Royal Anthropological Institute. 10: 395-420.

AZEVEDO, M.; A. BRAND; A. M. GOROSITO; E. HECK; B. MELIÁ \& J. SERVÍN. 2009. Guaraní Retã 2008, los pueblos guaraníes en las fronteras, Argentina, Brasil y Paraguay. B. Meliá (Ed.), AGR servicios gráficos, Asunción, $23 \mathrm{pp}$.

BALÉE, W. 1994. Footprints of the Forest. Ka'apor Ethnobotany, The Historical Ecology of Plant Utilization by an Amazonian People. Ed. Columbia University Press. New York.

— \& M. CEBOLLA BADIE. 2009. The Meaning of "Tree" in Two Different Tupí-Guaraní Languages from Two Different Neotropical ForestsAmazônica 1 (1): 96-135.

BARBOZA G. E., J. J. CANTERO, C. NÚÑEZ, A. PACCIARONI \& L. ARIZA ESPINAR. 2009. Medicinal plants: A general review and a phytochemical and ethnopharmacological screening of the native Argentine Flora. Kurtziana 34 (1-2): 7-365.

BARTOLOMÉ, M. A. 2009. Parientes de la selva, los guaraníes Mbya de la Argentina. Ed. CEADUC, Asunción, 463 pp.

BERLIN, B. 1973. Folk Systematics in Relation to Biological Classification and Nomenclature. Annual Review in Ecological Systems 4: 259-271.

BERTONI, M.S. 1940. Diccionario Botánico LatinoGuaraní; Guaraní-Latino. Ed. Guaraní. Asunción, $156 \mathrm{pp}$.

BROWN, C. H. 2000. Folk classification: an introduction. In. Paul E. Minis (Ed.): Ethnobotany, a reader. University of Oklahoma Press, Norman, p. 65-68.

CADOGAN, L. 1957. Breve contribución al estudio de la nomenclatura guaraní en Botánica. Ministerio de Agricultura y Ganadería. Servicio Técnico Interamericano de Cooperación Agrícola. Boletín nro. 194, Asunción, 49 pp.

1968. Chono Kybwyra: aporte al conocimiento de la mitología guaraní. Suplemento Antropológico de la Revista del Ateneo Paraguayo 3(1-2): 55-158.

CADOGAN, L. 1992. Diccionario Mbya-GuaraníCastellano. Biblioteca Paraguaya de Antropología, Asunción, 17: 1-211.

CAMARA HERNÁNDEZ, J. \& A. M. MIANTE ALZOGARAY. 2011. Razas de maíz nativas de Misiones. Boletín de la Sociedad Argentina de Botánica 46(Supl.): 231.

CEBOLLA BADIE, M. 2000. El conocimiento Mbya Guaraní de las aves. Nomenclatura y Clasificación. Suplemento Antropológico, Universidad Católica, Asunción. 35 (2): 9-188.

CHIARINI, C. H. B. 1975. La vuelta a los vegetales. Ed. Hachette, Buenos Aires, 631 pp.

CRIVOS, M., M. R. MARTÍNEZ, M. L. POCHETTINO,
C. REMORINI, A. SY \& L. TEVES. 2007. Pathways as "signatures in landscape": towards an ethnography of mobility among the Mbya-Guaraní (Northeastern Argentina). Journal of Ethnobiology and Ethnomedicine 3(2): 1-12.

DENNLER, J. G. 1939. Los nombres indígenas en guaraní de los mamíferos argentinos y países limítrofes y su importancia para la Sistemática. Physis 16(48): 225-244.

FAUSTO, C. 2005. Se deus fosse jaguar: canibalismo e cristianismo entre os guarani (séculos XVI-XX). Mana 11(2): 385-418.

GUASCH, A. \& D. ORTIZ. 1995. Diccionario Castellano-Guaraní, Guarani-Castellano. Sintáctico, fraseológico, ideológico. $12^{\circ}$ edición. C. E. P. A. G. Asunción. 826 pp.

GUBER, R. 2006. La etnografía, método, campo y reflexividad. Grupo editorial Norma. Buenos Aires, Bogotá, 146 pp.

GUPTA, M.P. 1995. Doscientas setenta plantas medicinales iberoamericanas. Ed. CYTED. Bogotá, 617 pp.

HVIDING, E. 2001. Naturaleza, cultura, magia, ciencia, sobre los metalenguajes de comparación en la Ecología Cultural. En: Descola, P. \& G. Pálsson. (Coords.). Naturaleza y Sociedad, perspectivas antropológica. Siglo XXI editores S.A. de C.V. México, p. 192-213.

HIERONYMUS, J. 1882. Planta diaphoricae florae argentinae. Academia Nacional de Ciencias, Córdoba 421 pp..

KELLER H. A. 2003. Mythical origin of Chusquea ramosissima Lindm. (Poaceae), the ancient knife of the Guaranis. Economic Botany 57 (4): 461-471.

. 2007a. Notas sobre medicina y magia entre los guaraníes de Misiones, Argentina, un enfoque etnobotánico. Suplemento Antropológico de la Universidad Católica de Asunción 42 (2): 345-384. . 2007b. Origen mítico de la yerba mate, Ilex paraguariensis A.St.-Hil. (Aquifoliaceae), una versión mbya guaraní. Suplemento Antropológico de la Universidad Católica de Asunción 42 (2): 335-344.

. 2009. Origen mítico del oso hormiguero (Myrmecophaga tridactyla), una versión Mbya guaraní. Suplemento Antropológico de la Universidad Católica. Asunción 44(2): 529-534. - 2010. Plantas colorantes de los guaraníes de Misiones, Argentina. Bonplandia 19(1): 11-25.

KOZEL, C. 1965. Salud y curación por yerbas. Ed. De la Misión, Buenos Aires, 1965.

LAHITTE, H. B. \& J, A. HURRELL. 1994. Las plantas de la medicina popular de la Isla Martín García (Nativas y naturalizadas), Reserva Natural y Cultural, Provincia de Buenos Aires, República Argentina. Comisión de Investigaciones Científicas (CIC). La Plata, 246 pp.

LEVI-STRAUSS, C. 1964. El pensamiento salvaje. 
Fondo de Cultura Económica. México, 413 pp.

MARTÍNEZ CROVETTO, R. N. 1968. Introducción a la Etnobotánica aborigen del nordeste argentino. Etnobiológica 11: 1-10.

—_ 1980. Plantas mágicas de los indios guaraníes de Misiones (República Argentina). La Antropología Americanista en la Actualidad, 2: 455-463

MARZOCCA, A. 1976. Manual de malezas. Ed. Hemisferio Sur. Buenos Aires, 564 pp.

METRAUX, A. 1948. The Guaraní. In Handbook of South American Indians, III. Washington.

MONTOYA, RUÍZ DE (S.J.). 1639. Tesoro de la lengua guaraní. Imprenta Juan Sánchez, Madrid, 814 pp.

MONTENEGRO, PEDRO DE (S. J.). 2007. Materia médica misionera. Editorial Universitaria de Misiones. Posadas, 313 pp.

MORA, V. V. 2008. Cuerpos, cadáveres y comida: canibalismo, comensalidad y organización social en la amazonía. Antípoda 6(1): 271-291.

MUIÑO, W. A., G. L. ALFONSO \& A. O. PRINA. 2006. Origen de los nombres vernáculos y usos de las plantas por parte de los pobladores del sur mendocino. Encuentro de Lenguas Indígenas Americanas, Santa Rosa 15-17 de Junio de 2006, 9 pp.

MÜLLER, F. 1989 [1935]. Etnografía de los Guaraní del Alto Paraná. Ed. Socieatis Verbi Divini, Rosario. $132 \mathrm{pp}$.

NEWMASTER, S. G., R. SUBRAMANYAN, R. F. IVANOFF \& N. C. BALASUBRAMANIAN. 2006. Mechanisms of ethnobiological classifications. Ethnobotany 18: 4-26.

OLIVEIRA D. DE. 2009. Nhanderukueri Ka'aguy Rupa - As florestas que pertencem aos deuses Etnobotânica e Territorialidade Guarani na Terra Indígena M'biguaçu/SC. Monografia apresentada como requisito parcial à obtenção do título de Bacharel em Ciências Biológicas, Centro de Ciência Biológicas, Universidade Federal de Santa Catarina. Florianópolis, $182 \mathrm{pp}$.

PLACCI, G. \& M. DI BITETTI. 2006. Situación ambiental en la ecorregión del Bosque Atlántico del Alto Paraná (Selva Paranaense), en Brown A., U. Martínez Ortíz, M. Acerbi \& J. Corcuera (eds.). La situación ambiental Argentina 2005. FVSArgentina. Buenos Aires, p. 193-210.

PODLECH, D. 2001. Herbs and healing plants of Britain \& Europe. London: Diamond Books. 249 pp.

QUEIREL, J. 1897. Misiones. Taller tipográfico de la Penitenciaría Nacional. Buenos Aires, p. 163.

RUIZ, H. 1952. Relación histórica del viage a los reynos del Peru y Chile. En J. Jaramillo-Arango (Ed.): Relación histórica del viage, que hizo a los reynos del Peru y Chile el botánico D. Hipólito Ruiz en el año de 1777 hasta el de 1788, en cuya época regreso a Madrid. Talleres gráficos de Cándido Bermejo, Madrid, 526 pp.

SCHMITZ, P.I. 1991. Migrantes da Amâzonia: A Tradição Tupiguaraní. En A. Kern et al.: Arqueologia pré-histórica do Rio Grande do Sul. P. 295-330. Porto Alegre.

STERNDALE-BENNETT, J. 2005. Plant names explained, botanical terms and their meaning. David \& Charles, Cincinnati, $224 \mathrm{pp}$.

STRASBURGER, E.; F. NOLL; H. SCHENCK \& A. F. W. SCHIMPER. 2003. Tratado de Botánica, $35^{\mathrm{a}}$ edición, actualizada por Sitte, P.; E. W. Weiler; J. W. Kadereit; A. Bresinsky \& C. Körner. Ediciones Omega, Barcelona, 1134 pp.

TASTEVIN, C, 1923. Nomes de plantas e Animaes em lingua Tupy. Separata do tomo XIII da revista do Museu Paulista, São Paulo. Officinas do "Diario Official", $77 \mathrm{pp}$.

TORRES MONTES, F. 1998. Fitónimos amerindios recogidos por José Celestino Mutis. Thesaurus 53(2): 242-270.

UTERMOEHL, B \& P. GONÇALVES. 2004. Conservação na roça (in situ) da agrobiodiversidade guaraní. Revista Brasileira de Agroecologia 2(1): 1708-1711.

VIVEIROS DE CASTRO, E. 1992. Os pronomes cosmológicos e o pespectivismo ameríndio. Mana 2(2): 115-144.

Original recibido el 9 de septiembre de 2011; aceptado el 17 de noviembre de 2011. 
Apéndice: Lista de las "plantas de los animales" según los guaraníes de Misiones, Argentina, especie, nombre guaraní, traducción, si son usadas en procedimientos mágicos (x), ejemplar testigo (Keller o Keller et al.) depositado en el herbario CTES del Instituto de Botánica del Nordeste.

\begin{tabular}{|c|c|c|c|c|}
\hline Especie & N. guaraní & Etimología & Mágica & Testigo \\
\hline \multicolumn{5}{|l|}{ MONOCOTILEDONEAE (LILIOPSIDA) } \\
\hline \multicolumn{5}{|l|}{ Araceae } \\
\hline Spathicarpa hastifolia Hook. & ta'ytetu ka'a & $\begin{array}{l}\text { hierba del pecarí de } \\
\text { collar }\end{array}$ & $\mathrm{x}$ & 1993 \\
\hline \multicolumn{5}{|l|}{ Commelinaceae } \\
\hline Commelina erecta $\mathrm{L}$. & kochi ka'a & hierba del pecarí labiado & $\mathrm{x}$ & 1141 \\
\hline Dichorisandra hexandra (Aubl.) Standl. & karaja ka'a & $\begin{array}{l}\text { hierba del mono } \\
\text { aullador }\end{array}$ & $\mathrm{x}$ & 694 \\
\hline \multicolumn{5}{|l|}{ Hydrocharitaceae } \\
\hline Egeria densa Planchon & pira ka'a & hierba del pez & $\mathrm{x}$ & 3395 \\
\hline \multicolumn{5}{|l|}{ Lemnaceae } \\
\hline Wolffia brasiliensis Wedd. & ju'y ka'a & hierba de la rana & $\mathrm{x}$ & 9610 \\
\hline \multicolumn{5}{|l|}{ Mayacaceae } \\
\hline Mayaca sellowiana Kunth. & karãu ka'a & hierba del ave carau & $\mathrm{x}$ & 833 \\
\hline \multicolumn{5}{|l|}{ Orchidaceae } \\
\hline Acianthera aveniformis Hoehne & kui'i ka'a & hierba del erizo & $\mathrm{x}$ & 3105 \\
\hline Acianthera recurva Lindl. & parakáu ka'a & hierba del loro hablador & $\mathrm{x}$ & ---- \\
\hline Anathallis linearifolia Cogn. & iñapeku ka'a & $\begin{array}{l}\text { hierba del pájaro } \\
\text { carpintero }\end{array}$ & $\mathrm{x}$ & 1430 \\
\hline Aspidogyne bruxellii (Pabst) Garay & inambu ka'a & hierba de la perdiz & $\mathrm{x}$ & 2024 \\
\hline Aspidogyne kuckzinskii (Porsch) Garay & inambu ka'a & hierba de la perdiz & $\mathrm{x}$ & 4922 \\
\hline Bulbophyllum regnellii Rchb. f.. & iñapeku ka'a & $\begin{array}{l}\text { hierba del pájaro } \\
\text { carpintero }\end{array}$ & $\mathrm{x}$ & 3533 \\
\hline Campylocentrum grisebachii Cogn. & ju'y ka'a & hierba de la rana & $\mathrm{x}$ & ---- \\
\hline Corymborkis flava (Sw.) Kuntze & mborevi ka'a & hierba del tapir & $\mathrm{x}$ & 8312 \\
\hline Cyclopogon congestus (Vell.) Hoehne & ñandu ka'a & hierba de la araña & & 1321 \\
\hline Cyclopogon elatus (Sw.) Schltr. & $\tilde{n} a n d u k a ' a$ & hierba de la araña & & 1142 \\
\hline Isabelia virginalis Barb. Rodr. & kavure'i ka'a & $\begin{array}{l}\text { hierba de la lechuza } \\
\text { caburé }\end{array}$ & $\mathrm{x}$ & ---- \\
\hline Sophronitis cernua Lindl. & parakáu ka'a & hierba del loro hablador & $\mathrm{x}$ & 741 \\
\hline \multicolumn{5}{|l|}{ Poaceae } \\
\hline Stenachaenium riedelii Baker & ambere ka'a & $\begin{array}{l}\text { hierba de la lagartija } \\
\text { amberé }\end{array}$ & $\mathrm{x}$ & 4812 \\
\hline
\end{tabular}

\section{DYCOTILEDONEAE (MAGNOLIOPSIDA)}

Acanthaceae

Justicia brasiliana Roth.

kochi ka'a

hierba del mono

141

Hydrocharitaceae

Egeria densa Planchon

pira ka'

395 
BONPLANDIA 20(2). 2011

\begin{tabular}{|c|c|c|c|c|}
\hline Especie & N. guaraní & Etimología & Mágica & Testigo \\
\hline Ruellia erythropus (Nees) Lindau & jeruty $k a^{\prime} a$ & $\begin{array}{l}\text { hierba de la paloma } \\
\text { yerutí }\end{array}$ & $\mathrm{x}$ & 3569 \\
\hline Ruellia geminiflora Kunth & guachu ka'a & hierba del venado & $\mathrm{x}$ & 5717 \\
\hline Stenandrium mandioccanum Nees. & peku'i ka'a & $\begin{array}{l}\text { hierba del pequeño } \\
\text { pájaro carpintero }\end{array}$ & $\mathrm{x}$ & 4412 \\
\hline \multicolumn{5}{|l|}{ Amaranthaceae } \\
\hline Iresine diffusa Humb. \& Bonpl. & $\begin{array}{l}\text { kavara ka'a } \\
\text { guachu }\end{array}$ & gran hierba de la cabra & $\mathrm{x}$ & 1360 \\
\hline \multicolumn{5}{|l|}{ Apiaceae } \\
\hline Bowelsia incana Ruiz \&Pav. & tatu ka'a & hierba del armadillo & $\mathrm{x}$ & 7496 \\
\hline Cyclospermum leptophyllum (Pers.) Sprague & jaguarẽ ka'a & hierba del zorrino & $\mathrm{x}$ & 7612 \\
\hline Hydrocotyle callicephala Cham. \& Schltdl. & jachyta ka'a & hierba del caracol & & 1850 \\
\hline Hydrocotyle exigua (Urb.) Malme & jachyta ka'a & hierba del caracol & & ---- \\
\hline Hydrocotyle leucocephala Cham. & jachyta ka'a & hierba del caracol & & 1179 \\
\hline \multicolumn{5}{|l|}{ Aristolochiaceae } \\
\hline Aristolochia elegans Mast. & ipe ka'a & hierba del pato & $\mathrm{x}$ & 5846 \\
\hline \multicolumn{5}{|l|}{ Asclepiadaceae } \\
\hline Asclepias curassavica $\mathrm{L}$. & mbói ka’a & hierba de la serpiente & $\mathrm{x}$ & 3538 \\
\hline Fischeria stellata (Vell.) E. Fourn. & yryvu ka'a & hierba del jote & $\mathrm{x}$ & 3779 \\
\hline Oxypetalum appendiculatum Mart. & mbóichy ka'a & $\begin{array}{l}\text { hierba d la vívora } \\
\text { mboychy }\end{array}$ & $\mathrm{x}$ & 3532 \\
\hline \multicolumn{5}{|l|}{ Asteraceae } \\
\hline Achyrocline tomentosa Rusby & jatei ka'a & $\begin{array}{l}\text { hierba del melipónido } \\
\text { yateí }\end{array}$ & $\mathrm{x}$ & 5038 \\
\hline Acanthospermum australe (Loefl.) Kuntze & eira viju ka'a & $\begin{array}{l}\text { hierba del melipónido } \\
\text { eiravijú }\end{array}$ & $\mathrm{x}$ & 5037 \\
\hline Baccharidiastrum triplinervium (Less.) Cabrera & kapi’igua ka'a & hierba del carpincho & & 1869 \\
\hline Baccharis brchylaenoides DC. & teju ka'a & hierba del lagarto & $\mathrm{x}$ & 323 \\
\hline Baccharis trimera (Less.) DC. & jaguarete ka'a & hierba del jaguar & $\mathrm{x}$ & 4104 \\
\hline Calea pinnatifida (R. BR.) Less. & jaku ka'a & $\begin{array}{l}\text { hierba de la pava de } \\
\text { monte }\end{array}$ & $\mathrm{x}$ & 8770 \\
\hline \multirow[t]{2}{*}{ Centratherum punctatum Cass. } & $a k a \tilde{e} k a^{\prime} a$ & hierba de la urraca & $\mathrm{x}$ & 2377 \\
\hline & urutu ka'a & $\begin{array}{l}\text { hierba de la serpiente } \\
\text { urutú }\end{array}$ & & \\
\hline Elephantopus mollis H.B.K. & pirincho ka'a & hierba del ave pilincho & $\mathrm{x}$ & 4741 \\
\hline Eupatorium caaguazense Choda. & pirincho ka'a & hierba del ave pilincho & $\mathrm{x}$ & 8277 \\
\hline Eupatorium filifolium Hassl. & karãu ka'a & hierba del ave carau & $\mathrm{x}$ & 8264 \\
\hline Eupatorium hecatanthum (DC.) Baker & $\begin{array}{l}\text { eiruchuka'a, } \\
\text { mandori ka'a }\end{array}$ & $\begin{array}{l}\text { hierba del melipónido } \\
\text { mandori }\end{array}$ & $\mathrm{x}$ & 7818 \\
\hline Eupatorium pseudoprassifolium Hassl. & eiraviju ka'a & $\begin{array}{l}\text { hierba del melipónido } \\
\text { eiraviju }\end{array}$ & $\mathrm{x}$ & 282 \\
\hline Eupatorium verbenaceum DC. & teju ka'a & hierba del lagarto & $\mathrm{x}$ & 4139 \\
\hline Galinsoga parviflora Cav. & eiru ka'a & hierba de la abeja & $\mathrm{x}$ & 3080 \\
\hline Lepidoploa balansae (Hieron.) H. Rob. & gajo ka'a, & hierba del gallo & $\mathrm{x}$ & 8792 \\
\hline Mikania cordifolia (L. f.) Willd. & aguara ka'a & hierba del zorro & & 2075 \\
\hline
\end{tabular}


H. A. Keller, Problemas de la etnotaxonomía guaraní: "Las plantas de los animales"

\begin{tabular}{|c|c|c|c|c|}
\hline Especie & N. guaraní & Etimología & Mágica & Testigo \\
\hline Mikania lindleyana DC. & $\begin{array}{l}\text { aguara ka'a } \\
\text { jaryi }\end{array}$ & hierba del zorro (débil) & & 1878 \\
\hline Mikania micrantha Kunth & aguara ka'a & hierba del zorro & & 654 \\
\hline Mutisia coccínea A. St.-Hil. & $\begin{array}{l}\text { guyra takuru } \\
\text { ka'a }\end{array}$ & hierba del ave chacurrú & $\mathrm{x}$ & 2582 \\
\hline Pluchea sagittalis (Lam.) Cabrera & mborevi ka'a & hierba del tapir & $\mathrm{x}$ & 4792 \\
\hline Podocoma rivularis (Gardner) G.L. Nesom & jaicha ka'a & hierba del agutí paca & $\mathrm{x}$ & 331 \\
\hline Pterocaulon alopecuroides (Lam.) DC. & $\begin{array}{l}\text { toro ka'a } \\
\text { morochĩ }\end{array}$ & hierba del toro & $\mathrm{x}$ & 4095 \\
\hline Pterocaulon balansae Chodat & $\begin{array}{l}\text { toro ka'a } \\
\text { morochĩ }\end{array}$ & hierba del toro & $\mathrm{x}$ & 1701 \\
\hline Pterocaulon lorentzii Malme & toro ka'a & hierba del toro & $\mathrm{x}$ & 8174 \\
\hline Pterocaulon polystachyum DC. & toro ka'a & hierba del toro & $\mathrm{x}$ & 2049 \\
\hline Smallanthus conatus (Spreng.) H. Rob. & jaguarete ka'a & hierba del jaguar & $\mathrm{x}$ & 3396 \\
\hline Soliva sessilis Ruiz \& Pav. & kui'ika'a & hierba del erizo & $\mathrm{x}$ & 1340 \\
\hline Vernonia brevifolia Less. & pynguarei ka'a & $\begin{array}{l}\text { hierba del melipónido } \\
\text { pinguareí }\end{array}$ & $\mathrm{x}$ & 4211 \\
\hline Vernonia tweediana Baker & jagua ka'a & hierba del perro & $\mathrm{x}$ & 720 \\
\hline \multicolumn{5}{|l|}{ Begoniaceae } \\
\hline Begonia cucullata Willd. & araku ka'a & hierba de la saracura & $\mathrm{x}$ & 2483 \\
\hline Begonia per-dusenni Brade & araku ka'a & hierba de la saracura & $\mathrm{x}$ & 2799 \\
\hline Begonia subvillosa Klotzsch & araku pyta ka'a & hierba del ipacaá & $\mathrm{x}$ & 327 \\
\hline \multicolumn{5}{|l|}{ Bignoniaceae } \\
\hline Dolichandra uncata (Adrews) L.G. Lohman & chivi ka'a & hierba del jaguar & $\mathrm{x}$ & ---- \\
\hline Dolichandra quadrivalvis (Jacq.) L. G. Lohman & $\begin{array}{l}\text { teju ka'a } \\
\text { ka'aguy }\end{array}$ & $\begin{array}{l}\text { hierba selvática del } \\
\text { lagarto }\end{array}$ & $\mathrm{x}$ & 2507 \\
\hline \multicolumn{5}{|l|}{ Boraginaceae } \\
\hline Varronia dichotoma Ruiz \& Pav. & $\begin{array}{l}\text { gajo ka'a } \\
\text { ka'aguy }\end{array}$ & hierba silvestre del gallo & $\mathrm{x}$ & 4119 \\
\hline Heliotropium indicum $\mathrm{L}$. & gajo ka'a & hierba del gallo & $\mathrm{x}$ & 8682 \\
\hline Heliotropium leiocarpum Morong & uru tuja ka'a & hierba del gallo & $\mathrm{x}$ & 400 \\
\hline Heliotropium salicoides Cham. & teju'ro'y ka'a & hierba de la lagartija & $\mathrm{x}$ & ---- \\
\hline Heliotropium transalpinum Vell. & uru tuja ka'a, & hierba del gallo & $\mathrm{x}$ & 7619 \\
\hline Tournefortia paniculata Cham. & urutau ka'a & hierba del ave urutaú & $\mathrm{x}$ & 1756 \\
\hline \multicolumn{5}{|l|}{ Brassicaceae } \\
\hline Coronopus didymus (L.) Sm. & jaguarẽ ka'a & hierba del zorrino & $\mathrm{x}$ & ---- \\
\hline \multicolumn{5}{|l|}{ Buddlejaceae } \\
\hline Buddleja stachyoides Cham. \& Schltdl. & toro ka'a jaryi & hierba del toro (débil) & $\mathrm{x}$ & 1110 \\
\hline \multicolumn{5}{|l|}{ Clusiaceae } \\
\hline Hypericum brasiliense Choisy & kavara ka'a & hierba de la cabra & $\mathrm{x}$ & 7649 \\
\hline \multicolumn{5}{|l|}{ Convolvulaceae } \\
\hline Dichondra macrocalyx Meissn. & $\begin{array}{l}\text { jachyta ka'a } \\
\text { jaryi }\end{array}$ & $\begin{array}{l}\text { hierba del caracol } \\
\text { (débil) }\end{array}$ & $\mathrm{x}$ & 293 \\
\hline
\end{tabular}




\begin{tabular}{|c|c|c|c|c|}
\hline Especie & N. guaraní & Etimología & Mágica & Testigo \\
\hline Dichondra microcalyx (Hallier f.) Fabris & uru ka'a & hierba del ave urú & $\mathrm{x}$ & 7521 \\
\hline Evolvulus sp. & anguja ka'a & hierba del ratón & $\mathrm{x}$ & 3665 \\
\hline Ipomoea quamoclit L. f. & mbore ka'a & hierba del tapir & $\mathrm{x}$ & 3409 \\
\hline \multicolumn{5}{|l|}{ Euphorbiaceae } \\
\hline Alchornea glandulosa Poepp. & chi'yka'a & hierba del coatí & $\mathrm{x}$ & 1304 \\
\hline Alchornea triplinervia (Spreng.) Müll. Arg. & $c h i ' y k a ' a$ & hierba del coatí & $\mathrm{x}$ & 1300 \\
\hline Euphorbia heterophylla L. & piku’i ka'a & hierba de la paloma & $\mathrm{x}$ & 2999 \\
\hline $\begin{array}{l}\text { Euphorbia papillosa A. St.-Hil. var. cabrerae } \\
\text { Subils }\end{array}$ & pira ka'a & hierba del pez & $\mathrm{x}$ & ---- \\
\hline Euphorbia serpens Kunth & jatevu ka'a & hierba de la garrapata & $\mathrm{x}$ & 6083 \\
\hline Phyllanthus caroliniensis Walter & ychau ka'a & $\begin{array}{l}\text { hierba de la hormiga } \\
\text { cortadora }\end{array}$ & $\mathrm{x}$ & 151 \\
\hline \multicolumn{5}{|l|}{ Fabaceae } \\
\hline $\begin{array}{l}\text { Chamaecrista desvauxii (Collad.) Killip var. } \\
\text { mollissima (Benth.) Irwin \& Barneby }\end{array}$ & tanambi ka'a & hierba de la polilla & $\mathrm{x}$ & ---- \\
\hline Collaea stenophylla (Hook. et Arn.) Benth. & piky ka'a & hierba de la mojarra & $\mathrm{x}$ & 7955 \\
\hline Desmodium affine Schlecht. & guachu ka'a & hierba del venado & $\mathrm{x}$ & 4511 \\
\hline Eriosema sp. & pirincho ka'a & hierba del ave pilincho & $\mathrm{x}$ & 5669 \\
\hline Melilotus albus Desv. & teju ka'a & hierba del lagarto & $\mathrm{x}$ & 4424 \\
\hline Poiretia latifolia Vogel & ychau ka'a & $\begin{array}{l}\text { hierba de la hormiga } \\
\text { cortadora }\end{array}$ & $\mathrm{x}$ & 4841 \\
\hline Rhynchosia lineata Benth. & guachu ka'a & hierba del venado & $\mathrm{x}$ & --- \\
\hline $\begin{array}{l}\text { Vigna peduncularis (Kunth) Fawc. \& Rendle } \\
\text { var. clitorioides (Benth.) Maréchal } \\
\text { Indeterminada }\end{array}$ & роро ka'a & hierba de la mariposa & $\mathrm{x}$ & 5003 \\
\hline Gen indet. 1 & kuchiu ka'a & $\begin{array}{l}\text { hierba del ave } \\
\text { chupadientes }\end{array}$ & $\mathrm{x}$ & ---- \\
\hline \multicolumn{5}{|l|}{ Lamiaceae } \\
\hline Buchnera integrifolia Larrañaga & piky ka'a & hierba de la mojarra & $\mathrm{x}$ & 4830 \\
\hline Hyptis althaefolia Pohl & taguato ka'a & $\begin{array}{l}\text { hierba de la rapaz } \\
\text { taguató }\end{array}$ & $\mathrm{x}$ & 5309 \\
\hline \multirow[t]{2}{*}{ Hyptis mutabilis (Rich.) Briq. } & \multirow{2}{*}{$\begin{array}{l}\text { pinguãre'i ka'a } \\
\text { taguato ka'a }\end{array}$} & $\begin{array}{l}\text { hierba del melipónido } \\
\text { pinguãrei }\end{array}$ & & 1715 \\
\hline & & hierba del ave taguató & & \\
\hline Leonurus japonicus Houtt. & kave'ẽ ka'a & hierba de la avispa & $\mathrm{x}$ & 2494 \\
\hline Ocimum selloi Benth. & mbói chini ka'a & $\begin{array}{l}\text { hierba de la serpiente de } \\
\text { cascabel }\end{array}$ & $\mathrm{x}$ & 6018 \\
\hline Salvia guaranitica Benth. & mbói ka'a & hierba de la serpiente & $\mathrm{x}$ & 2983 \\
\hline \multicolumn{5}{|l|}{ Lythraceae } \\
\hline Cuphea linarioides Cham. \& Schltdl. & mbói ka'a & hierba de la serpiente & $\mathrm{x}$ & 4474 \\
\hline Heimia salicifolia (Kunth) Link & karaja ka'a & $\begin{array}{l}\text { hierba del mono } \\
\text { aullador }\end{array}$ & $\mathrm{x}$ & 7717 \\
\hline \multicolumn{5}{|l|}{ Malvaceae } \\
\hline Pavonia communis A. St.-Hil. & $k a^{\prime} i k a^{\prime} a$ & hierba del mono & $\mathrm{x}$ & 6428 \\
\hline Pavonia sepium A. St.-Hil. & mbutu ka'a & hierba del tábano & $\mathrm{x}$ & 7625 \\
\hline
\end{tabular}


H. A. Keller, Problemas de la etnotaxonomía guaraní: "Las plantas de los animales"

\begin{tabular}{|c|c|c|c|c|}
\hline Especie & N. guaraní & Etimología & Mágica & Testigo \\
\hline Pavonia subrotunda A. St.-Hil. \& Maudin & $k a^{\prime} i k a^{\prime} a$ & hierba del mono & $\mathrm{x}$ & 5802 \\
\hline Wissadula glechomatifolia (A. St.-Hil.) R. E. Fr. & jate’ $i k a ' a$ & $\begin{array}{l}\text { hierba del melipónido } \\
\text { yateí }\end{array}$ & $\mathrm{x}$ & 8129 \\
\hline Sida urens L. & tapichí ka'a & hierba del conejo & $\mathrm{x}$ & 658 \\
\hline \multicolumn{5}{|l|}{ Malpighiaceae } \\
\hline Galphimia australis Chodat & churuku'a ka'a & hierba del surucuá & $\mathrm{x}$ & 4547 \\
\hline \multicolumn{5}{|l|}{ Martynianecae } \\
\hline Craniolaria integrifolia Cham. & jatevu ka'a ñu & $\begin{array}{l}\text { hierba campestre de la } \\
\text { garrapata }\end{array}$ & $\mathrm{x}$ & 9486 \\
\hline \multirow[t]{2}{*}{ Ibicella lutea (Lindl.) Van Eselt. } & jatevu ka'a & hierba de la garrapata & $\mathrm{x}$ & ---- \\
\hline & mbói ka'a & hierba de la serpiente & & \\
\hline \multicolumn{5}{|l|}{ Moraceae } \\
\hline Dorstenia brasiliensis Lam. & kavure ka'a & $\begin{array}{l}\text { hierba de la lechuza } \\
\text { caburé }\end{array}$ & $\mathrm{x}$ & 398 \\
\hline \multicolumn{5}{|l|}{ Myrsinaceae } \\
\hline Myrsine balansae (Mez) Otegui & apere'a ka'a & hierba del cuis & $\mathrm{x}$ & 1362 \\
\hline \multicolumn{5}{|l|}{ Oxalidaceae } \\
\hline Oxalis sexeneta Sav. & kavure'i ka'a & $\begin{array}{l}\text { hierba de la lechuza } \\
\text { caburé }\end{array}$ & $\mathrm{x}$ & 5859 \\
\hline \multicolumn{5}{|l|}{ Passifloraceae } \\
\hline Passiflora alata Dryand. & jaku ka'a & $\begin{array}{l}\text { hierba de la pava de } \\
\text { monte }\end{array}$ & $\mathrm{x}$ & 8728 \\
\hline Passiflora capsularis L. & tapẽ $k a$ 'a & $\begin{array}{l}\text { hierba del milano } \\
\text { tijereta }\end{array}$ & $\mathrm{x}$ & 2579 \\
\hline Passiflora edulis Sims. & guachu ka'a & hierba del venado & $\mathrm{x}$ & 4395 \\
\hline \multicolumn{5}{|l|}{ Piperaceae } \\
\hline Peperomia aceroana C.DC. & jatevu ka'a & hierba de la garrapata & & 464 \\
\hline Peperomia balansana C.DC. & uru ka'a & hierba del ave urú & $\mathrm{x}$ & 1842 \\
\hline Peperomia blanda (Jacq.) Kunth & churuku'a ka'a & hierba del ave surucuá & $\mathrm{x}$ & 5072 \\
\hline Peperomia catharinae Miq. & jatevu ka'a & hierba de la garrapata & & 5892 \\
\hline Peperomia circinata Link & jatevu ka'a & hierba de la garrapata & & 7728 \\
\hline Peperomia corcovadensis Gardner & uru ka'a & hierba del ave urú & $\mathrm{x}$ & 1998 \\
\hline Peperomia delicatula Henschen & jatevu ka'a & hierba de la garrapata & & 239 \\
\hline Peperomia hilariana Miq. & churuku'a ka'a & hierba del ave surucuá & $\mathrm{x}$ & 5057 \\
\hline Peperomia hispidula (Sw.) A. Dietr. & uru ka'a & hierba del ave urú & $\mathrm{x}$ & 5058 \\
\hline Peperomia rotundifolia (L.) Kunth & jatevu ka'a & hierba de la garrapata & & 2237 \\
\hline Peperomia rubricaulis (Nees) A. Dietr. & $\begin{array}{l}\text { churuku'a pytã } \\
\text { ka'a }\end{array}$ & $\begin{array}{l}\text { hierba del ave surucuá } \\
\text { rojo }\end{array}$ & $\mathrm{x}$ & 3436 \\
\hline Peperomia tetraphylla (G. Forst.) Hook. et Arn. & uru ka'a & hierba del ave urú & $\mathrm{x}$ & 1887 \\
\hline Peperomia trapezoidalis Yunck. & jatevu ka'a & hierba de la garrapata & & 2733 \\
\hline Peperomia urocarpa Fisch. \& C.A. Mey. & $\begin{array}{l}\text { churuku'a ka'a } \\
\text { jaryi }\end{array}$ & $\begin{array}{l}\text { hierba débil del ave } \\
\text { surucuá }\end{array}$ & $\mathrm{x}$ & 262 \\
\hline Piper amalago $\mathrm{L}$. & jakare ka'a & hierba del caimán & $\mathrm{x}$ & 2730 \\
\hline
\end{tabular}




\begin{tabular}{|c|c|c|c|c|}
\hline Especie & N. guaraní & Etimología & Mágica & Testigo \\
\hline \multicolumn{5}{|l|}{ Plantaginaceae } \\
\hline Plantago tomentosa Lam. & uru ka'a & hierba del ave urú & $\mathrm{x}$ & 3723 \\
\hline \multicolumn{5}{|l|}{ Portulacaceae } \\
\hline Portulaca cryptopetala Speg. & $\begin{array}{l}\text { churuku'a ka'a } \\
\text { mirĩ }\end{array}$ & $\begin{array}{l}\text { hierba pequeña del ave } \\
\text { surucuá }\end{array}$ & $\mathrm{x}$ & 1658 \\
\hline \multicolumn{5}{|l|}{ Proteaceae } \\
\hline $\begin{array}{l}\text { Roupala montana Aubl.var. brasiliensis } \\
\text { (Klotzsch) K.S. Edwards }\end{array}$ & $\begin{array}{l}\text { mborevi ka'a } \\
\text { guachu }\end{array}$ & gran hierba del tapir & $\mathrm{x}$ & 4117 \\
\hline \multicolumn{5}{|l|}{ Rubiaceae } \\
\hline Borreria verticillata (L.) Mey. & eira viju ka'a & $\begin{array}{l}\text { hierba del melipónido } \\
\text { eiraviju }\end{array}$ & $\mathrm{x}$ & 384 \\
\hline Faramea cyanea Müll. Arg. & mborevi ka'a & hierba del tapir & $\mathrm{x}$ & 8769 \\
\hline $\begin{array}{l}\text { Galianthe brasiliensis (Spreng.) E.L. Cabral \& } \\
\text { Bacigalupo }\end{array}$ & piky ka'a & hierba de la mojarra & $\mathrm{x}$ & 8146 \\
\hline Galium latoramosum Clos & anguja ka'a & hierba del ratón & $\mathrm{x}$ & 2414 \\
\hline $\begin{array}{l}\text { Galium richardianum (Gillies ex Hook. \&Arn.) } \\
\text { Endl. exWalp. }\end{array}$ & anguja ka'a & hierba del ratón & $\mathrm{x}$ & 2173 \\
\hline Geophila repens (L.) Johnston & uru'ika'a & hierba del ave urú & $\mathrm{x}$ & 5020 \\
\hline Ixora venulosa Benth. & tapichĩ ka'a & hierba del conejo & $\mathrm{x}$ & 1370 \\
\hline Manettia luteo rubra (Vel.) Benth. & maino ka'a & hierba del colibrí & $\mathrm{x}$ & 7629 \\
\hline Psychotria brevicollis Müll. Arg. & tapichĩ ka'a yrũ & $\begin{array}{l}\text { compañero de la hierba } \\
\text { del conejo }\end{array}$ & $\mathrm{x}$ & 2002 \\
\hline Psychotria carthagenensis Jacq. & $\begin{array}{l}\text { tapichĩ ka'a } \\
\text { guachu }\end{array}$ & gran hierba del conejo & $\mathrm{x}$ & 7981 \\
\hline Psychotria leiocarpa Cham. \&Schltdl. & tapichĩ ka'a & hierba del conejo & $\mathrm{x}$ & 1856 \\
\hline Psychotria myriantha Muell. Arg. & tapichĩ ka'a & hierba del conejo & $\mathrm{x}$ & 3279 \\
\hline Psychotria suturella Müll. Arg. & $\begin{array}{l}\text { tapichĩ ka'a } \\
\text { guachu }\end{array}$ & gran hierba del conejo & $\mathrm{x}$ & 9382 \\
\hline Richardia brasiliensis Gomes & $\tilde{n} a n d u k a ' a$ & hierba de la araña & & 2200 \\
\hline Staelia thymoides Cham. Et Schltdl. & eira viju ka'a & $\begin{array}{l}\text { hierba del melipónido } \\
\text { eiraviju }\end{array}$ & $\mathrm{x}$ & 5667 \\
\hline \multicolumn{5}{|l|}{ Sapindaceae } \\
\hline $\begin{array}{l}\text { Cardiospermum halicacabum L. var. } \\
\text { microcarpum (Kunth) Blume }\end{array}$ & guachu ka'a & hierba del venado & $\mathrm{x}$ & 6090 \\
\hline \multicolumn{5}{|l|}{ Scrophulariaceae } \\
\hline Agalinis genistifolia (Cham. \& Schltd1.) D'Arcy & arapachái ka’a & hierba del loro maitaca & $\mathrm{x}$ & ---- \\
\hline Angelonia integerrima Spreng. & karaja ka'a & $\begin{array}{l}\text { hierba del mono } \\
\text { aullador }\end{array}$ & $\mathrm{x}$ & 4845 \\
\hline Mecardonia tenella Cham. & ju'y ka'a & hierba de la rana & $\mathrm{x}$ & 347 \\
\hline Scoparia montevidensis (Spreng.) R.R.Fr. & $\begin{array}{l}\text { uru ka'a } \\
\text { ka'aguy }\end{array}$ & $\begin{array}{l}\text { hierba selvática del ave } \\
\text { urú }\end{array}$ & $\mathrm{x}$ & 77 \\
\hline Stemodia verticillata (Mill.) Hassl. & takuru ka'a & hierba del ave chacurrú & $\mathrm{x}$ & 3530 \\
\hline \multicolumn{5}{|l|}{ Solanaceae } \\
\hline Capsicum flexuosum Sendtn. & guachu ka'a & hierba del venado & $\mathrm{x}$ & 1144 \\
\hline Cyphomandra scyadostilis Sendtn. & chavapẽ ka'a & hierba del hurón & $\mathrm{x}$ & 2578 \\
\hline
\end{tabular}


H. A. Keller, Problemas de la etnotaxonomía guaraní: "Las plantas de los animales"

\begin{tabular}{|c|c|c|c|c|}
\hline Especie & N. guaraní & Etimología & Mágica & Testigo \\
\hline \multirow[t]{2}{*}{ Cyphomandra corymbiflora Sendtn. } & kururu ka'a & hierba del sapo & $\mathrm{x}$ & 286 \\
\hline & yryvu ka'a & hierba del jote & & \\
\hline Petunia inflata R. E. Fr. & $\underset{\text { pytã }}{\operatorname{inambu} k a^{\prime} a}$ & hierba de la perdiz roja & $\mathrm{x}$ & 4440 \\
\hline Solanum inaequale Vell. & paroma ka'a & $\begin{array}{l}\text { hierba de la paloma } \\
\text { doméstica }\end{array}$ & $\mathrm{x}$ & 792 \\
\hline \multicolumn{5}{|l|}{ Sterculiaceae } \\
\hline Melochia pilosa (Mill.) Fawc \& Rendl. & $\tilde{n} a n d u k a^{\prime} a$ & hierba de la araña & $\mathrm{x}$ & 434 \\
\hline \multicolumn{5}{|l|}{ Valerianaceae } \\
\hline Valeriana scandens Loefl. ex. L. & kochi ka'a & hierba del pecarí labiado & $\mathrm{x}$ & 1061 \\
\hline \multicolumn{5}{|l|}{ Verbenaceae } \\
\hline Lantana brasiliensis Link. & eirachĩ ka'a & $\begin{array}{l}\text { hierba del melipónido } \\
\text { eirachî }\end{array}$ & $\mathrm{x}$ & 55 \\
\hline Lippia lippioides (Cham.) Rusby & jagua ka'a & hierba del perro & $\mathrm{x}$ & 3453 \\
\hline Lippia lupulina Cham. & gajo ka'a & hierba del gallo & $\mathrm{x}$ & 5671 \\
\hline Peltodon longipes Benth. & tatu ka'a & hierba del armadillo & $\mathrm{x}$ & 7907 \\
\hline \multicolumn{5}{|l|}{ Violaceae } \\
\hline Hybanthus parviflorus (Mutis ex L. f.) Baill & aguara'i ka'a & hierba del zorrito & $\mathrm{x}$ & 3483 \\
\hline \multicolumn{5}{|l|}{ Viscaceae } \\
\hline Phoradendron falcifrons (Hook, \& Arn.) Eichler & ypeku ka'a & $\begin{array}{l}\text { hierba del pájaro } \\
\text { carpintero }\end{array}$ & $\mathrm{x}$ & 2031 \\
\hline Phoradendron piperoides (Kunth) Nutt. & karaja ka'a & $\begin{array}{l}\text { hierba del mono } \\
\text { aullador }\end{array}$ & $\mathrm{x}$ & 3471 \\
\hline
\end{tabular}

\section{PTERIDOPHYTA}

\section{Lycopodiaceae}

Huperzia mandioccana (Raddi) Trevis

karau ka'a
ka'aguy
karau ka'a
urukure'aka'a

hierba selvática del ave

caraú

Lycopodiella cernua (L.) Pic. Serm.

hierba del ave caraú

Lycopodium clavatum L.

hierba del búho

9587

\section{Polypodiaceae}

Campyloneurum austrobrasilianum (Alston) de la Sota

manjy $\mathrm{ka}^{\prime} \mathrm{a}$

hierba de la lagartija

$\mathrm{x}$

49

Campyloneurum major (Hieron. ex Hicken)

Lellinger

Microgramma linbergii (Mett. ex Kuhn) de la

Sota

Microgramma squamulosa (Kaulf.) de la Sota

mburika ka'a

hierba del burro

$\mathrm{x}$

329

ambere $k a$ 'a

hierba de la lagartija

$\mathrm{x}$

1081

Microgramma vaccinifolia (Langsd. \& Fisch.)

Copel.

ambere ka'a amberé

$\mathrm{X}$

5678

hierba de la lagartija amberé

ambere ka'a

hierba de la lagartija amberé

Schyzaceae

Anemia simplicior (H. Christ) Mickel

jakare ka'a hierba del caimán x

4031

Anemia tomentosa (Savigny) Sw.

jakare ka'a mirĩ pequeña hierba del

$\mathrm{x}$

1639 
BONPLANDIA 20(2). 2011

\begin{tabular}{|c|c|c|c|c|}
\hline Especie & N. guaraní & Etimología & Mágica & Testigo \\
\hline Lygodium volubile $\mathrm{L}$. & jakare ka'a & hierba del caimán & $\mathrm{x}$ & 5814 \\
\hline \multicolumn{5}{|l|}{ Thelypteridaceae } \\
\hline Thelypteris riograndensis (Lindm.) C.F. Reed & jakare ka'a & hierba del caimán & $\mathrm{x}$ & 3439 \\
\hline
\end{tabular}

\section{BRYOPHYTA}

Familia indet.

Musci indet.

Marchantiaceae

Gén. Indet.

kavure ka'a

hierba de la lechuza

$\mathrm{x}$

808

LICHEN

Lichen indet. 1

Lichen indet. 2

karumbe ka'a

hierba de la tortuga

$\mathrm{X}$

FUNGI

Fungi indet. (con fosforescencia nocturna)

jatevu ka'a

hierba de la garrapata

$\mathrm{X}$

jaku ka'a

hierba de la pava de monte

X

manjy ka'a

hierba de la lagartija manyí

$\mathrm{X}$

----

